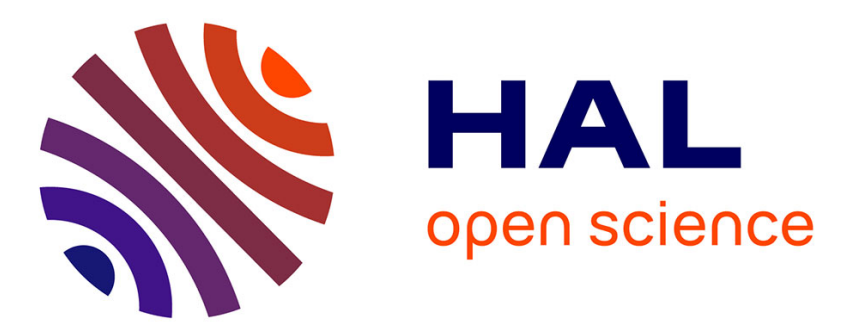

\title{
Closed-form solutions for the free-vibration problem of multilayered piezoelectric shells
}

\author{
M. d'Ottavio, D. Ballhause, B. Kröplin, E. Carrera
}

\section{To cite this version:}

M. d'Ottavio, D. Ballhause, B. Kröplin, E. Carrera. Closed-form solutions for the free-vibration problem of multilayered piezoelectric shells. Computers \& Structures, 2006, 84 (22-23), pp.1506-1518. 10.1016/j.compstruc.2006.01.030 . hal-01367083

\section{HAL Id: hal-01367083 \\ https://hal.science/hal-01367083}

Submitted on 10 Jan 2019

HAL is a multi-disciplinary open access archive for the deposit and dissemination of scientific research documents, whether they are published or not. The documents may come from teaching and research institutions in France or abroad, or from public or private research centers.
L'archive ouverte pluridisciplinaire HAL, est destinée au dépôt et à la diffusion de documents scientifiques de niveau recherche, publiés ou non, émanant des établissements d'enseignement et de recherche français ou étrangers, des laboratoires publics ou privés. 


\title{
Closed-form solutions for the free-vibration problem of multilayered piezoelectric shells
}

\author{
M. D’Ottavio, D. Ballhause, B. Kröplin, E. Carrera
}

\section{Introduction}

Piezoelectric components have been an important research object since many years due to their unique feature to couple electric and mechanical characteristics in one single material. For the last 50 years, their use as electro-mechanical transducers in sensor as well as in actuator applications has been continuously increasing. More recently, piezoelectrics have been considered among the most suitable materials for extending the structural capabilities beyond the purely passive load carrying one. Adaptive structures, in which the active material is embedded into the host structure, thus contributing to the overall stiffness, are an example for these striven high-performance structures. Vibration and noise suppression, controlled active deformation and health monitoring are among the

\footnotetext{
* Corresponding author. Tel.: +49 711 68562484; fax: +49 711 68563706.

E-mail address: dottavio@isd.uni-stuttgart.de (M. D’Ottavio).
}

most important applications of these "intelligent" structural components.

Since the typical structural elements in many engineering fields are thin, lightweight panels, the interest in twodimensional models able to predict the behavior of plates and shells with embedded piezoelectric elements have captured the attention of the research community. The introduction of piezoelectric material into a passive structure naturally leads to a multilayered component, and it has been recognized that classical models are not suitable for an accurate design of such structures, see for example the review article of Noor and Burton [1] and the references cited herein. Interlaminar Continuity (IC) of the transverse stresses as well as the related discontinuity of the slopes of the displacement distributions in thickness direction at the layers' interfaces are the main effects arising in multilayered structures which cannot be captured by classical formulations based on Love First Approximation Theories (LFAT), see e.g. [2]. Many refined theories for plates and shells have been proposed in order to meet the modeling 
requirements - known as $C_{z}^{0}$-Requirements - posed by these characteristics; further details can be found, e.g., in the monograph of Reddy [3] and in the paper of Carrera [4]. For curved structures, Koiter [5] recognized the importance of transverse stress effects even for homogeneous shells and recommended the inclusion of such effects whenever a consistent higher-order model has to be proposed.

The fundamentals for the modeling of piezoelectric materials have been given in many contributions, in particular in the pioneering works of Mindlin [6], EerNisse [7], Tiersten and Mindlin [8], and in the monograph of Tiersten [9]. The embedding of piezoelectric layers into plates and shells sharpens the requirements of an accurate modeling of the resulting adaptive structure due to the localized electro-mechanical coupling, see e.g. the review of Saravanos and Heyliger [10]. Therefore, within the framework of two-dimensional approaches, layerwise descriptions have been often proposed either for the electric field only (see, e.g., the works of Kapuria [11] and of Ossadzow-David and Touratier [12]) or for both the mechanical and electrical unknowns (e.g., [13]). An attempt to mathematically substantiate axiomatic two-dimensional piezoelectric shell formulations by the means of asymptotic expansions can be found in the book of Rogacheva [14]. An exhaustive overview of the many different modeling approaches and solution techniques for laminated piezoelectric plates and shells is far beyond the scope of this paper; more details on this topic can be found, e.g., in the already cited review of Saravanos and Heyliger, and in the surveys of Gopinathan et al. [15] and of Benjeddou [16]. For the last years interest has been emerging for mixed formulations involving also stresses and dielectric displacements as primary variables, see for example [17] and [18].

In the present work, a series of two-dimensional, hierarchic models for laminated shells including piezoelectric layers is presented and employed for the free-vibration analysis of shell-type structures. Linear up to fourth-order assumptions for the mechanical displacements and for the electric potential in the thickness direction have been considered. The number of mechanical unknowns can be independent from the number of layers constituting the laminate (Equivalent Single Layer - ESL - descriptions), or they can be defined in each layer (Layerwise - LW descriptions). The electric potential has always been considered within an LW description. The so-called "electric stiffness" of the piezoelectric layers can therefore be taken into account provided an at least linear variation of the electric field is assumed. The large number of different models could be conveniently implemented exploiting the "Unified Formulation" proposed by Carrera [19]. For certain mechanical and geometrical properties of the considered problems, a Navier-type closed-form solution can be found: this analytical approach fulfills exactly both the domain equations and the boundary conditions. This solution technique has already been successfully employed in combination with the Unified Formulation for elastic plates [20], elastic shells [21] and for piezoelectric plates
[22]. In all cases, the exact 3D solution (when available) could be recovered by the most accurate formulations. In this work, the modeling method based on the Unified Formulation and the analytical Navier-type solution is extended to doubly curved, layered shells embedding piezoelectric material. A good agreement with results available in open literature confirms the validity of the method also for piezoelectric shells. The presented formulations are exemplarily applied to the study of the influence of the electro-mechanical coupling on some characteristic vibratory behaviors of laminated cylinders.

The work is organized as follows: Section 2 presents the fundamental equations for the description of the shell geometry and kinematics as well as of the coupled electro-mechanical material behavior. The two-dimensional modeling is described in Section 3 and the resulting governing equations are derived in Section 4. Section 5 is dedicated to the numerical verification of the proposed model and to the analysis of the electro-mechanical coupling in adaptive shells.

\section{Preliminaries}

\subsection{Shell geometry}

The description of the geometry of the shell structure employed throughout this work is based on the classical works of Kraus [23] and Novozhilov [24]. In the following, the mathematical description of the multilayered shell geometry depicted in Fig. 1 will be given for each layer $k$. Thus, the index $k$ will in general not be a summation index. The reference surface $\Omega_{k}$ of the $k$ th layer is described with the curvilinear coordinates $\alpha_{k}, \beta_{k}$ which are lines of principal curvature, see Fig. 1. This reference system is always orthogonal since the directions of principal curvature are always mutually perpendicular. The increase of the arc length $\mathrm{d} s_{\alpha_{k}}$ corresponding to an infinitesimal increase $\mathrm{d} \alpha_{k}$ of the coordinate $\alpha_{k}$ defines the metric of the surface measured with the Lamé parameter $A_{k}$ according to

$\mathrm{d} s_{\alpha_{k}}=A_{k} \mathrm{~d} \alpha_{k}$

For the coordinate $\beta_{k}$ holds an analogous relation with the Lamé coefficient $B_{k}$. The thickness of the layer is measured

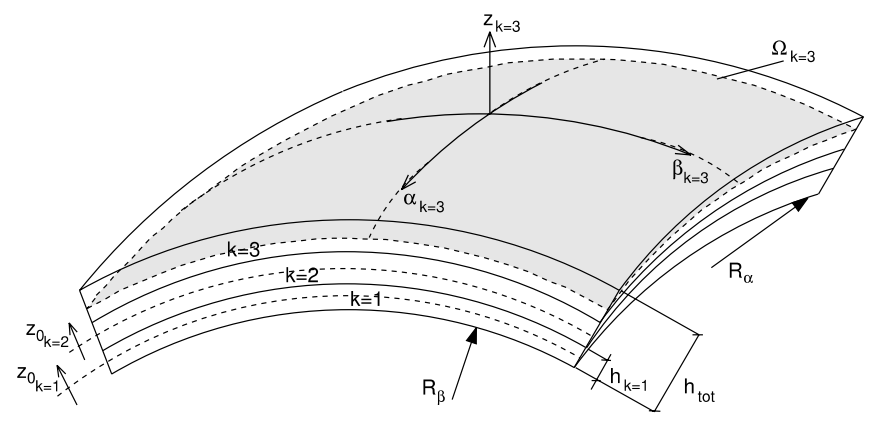

Fig. 1. Notations for the description of the shell geometry. 
along the direction perpendicular to the two curvilinear coordinates. In each point of the reference surface this direction is described by the rectilinear coordinate $z_{k}$; the Lamé coefficient associated to this coordinate is unitary. Considering the change of the radii of curvatures due to a variation along the thickness coordinate, the square of an infinitesimal linear segment in the layer is given by

$$
\left(\mathrm{d} s_{k}\right)^{2}=H_{\alpha_{k}}^{2}\left(\mathrm{~d} \alpha_{k}\right)^{2}+H_{\beta_{k}}^{2}\left(\mathrm{~d} \beta_{k}\right)^{2}+\left(\mathrm{d} z_{k}\right)^{2}
$$

with the two metric coefficients $H_{\alpha_{k}}, H_{\beta_{k}}$ given by

$H_{\alpha_{k}}=A_{k}\left(1+\frac{z_{k}}{R_{\alpha_{k}}}\right) ; \quad H_{\beta_{k}}=B_{k}\left(1+\frac{z_{k}}{R_{\beta_{k}}}\right)$

$R_{\alpha_{k}}$ and $R_{\beta_{k}}$ are the principal radii of curvature along the coordinates $\alpha_{k}$ and $\beta_{k}$, respectively. The associated infinitesimal area and volume elements are given accordingly by

$$
\begin{aligned}
\mathrm{d} \Omega_{k} & =H_{\alpha_{k}} H_{\beta_{k}} \mathrm{~d} \alpha_{k} \mathrm{~d} \beta_{k} \\
\mathrm{~d} V_{k} & =H_{\alpha_{k}} H_{\beta_{k}} \mathrm{~d} \alpha_{k} \mathrm{~d} \beta_{k} \mathrm{~d} z_{k}
\end{aligned}
$$

respectively. While the above equations hold for arbitrary shell geometries, in the remainder of this paper we restrict our analysis to shells with constant radii of curvature, i.e. with $A_{k}=B_{k}=1$. Cylindrical shells as well as open spherical panels can be included in the analysis. Closed spheres and general shells of revolution, for which the radius of curvature is a function of the distance from the axis are not accounted for.

\subsection{Geometric relations}

The strains arising in the shell after deformation are split in the in-plane and the transverse components, denoted $\left(\epsilon_{p}\right)_{i}$ and $\left(\epsilon_{n}\right)_{i}$, respectively. To the former belong the strains along the two curvilinear coordinates as well as the inplane shear deformation. The transverse strains are the components of the strain tensor related to a deformation in the thickness direction, i.e. the two transverse shear strains and the transverse direct strain. Within the small strain assumption, the linear geometrical relationships between the displacements $u_{i}^{k}=\left[u_{\alpha}^{k} ; u_{\beta}^{k} ; u_{z}^{k}\right]^{\mathrm{T}}$ and the strains in each layer $k$ read in compact array notation [23]

$\left(\epsilon_{p}\right)_{i}^{k}=\left[\epsilon_{\alpha \alpha}^{k}, \epsilon_{\beta \beta}^{k}, \epsilon_{\alpha \beta}^{k}\right]^{\mathrm{T}}=\left(D_{p}\right)_{i j} u_{j}^{k}+\left(A_{p}\right)_{i j} u_{j}^{k}$

$\left(\epsilon_{n}\right)_{i}^{k}=\left[\epsilon_{\beta z}^{k}, \epsilon_{\alpha z}^{k}, \epsilon_{z z}^{k}\right]^{\mathrm{T}}=\left(D_{n \Omega}\right)_{i j} u_{j}^{k}+\left(A_{n}\right)_{i j} u_{j}^{k}+\left(D_{n z}\right)_{i j} u_{j}^{k}$

where the usual summation convention has been employed and the superscript $\mathrm{T}$ indicates the transposition operator. The indices $i$ and $j$ are intended to run from 1 to 3 . The matrix operators in Eq. (5a) are split into a differential one and an operator related to the curvature terms:

$$
\left(D_{p}\right)_{i j}=\left[\begin{array}{ccc}
\frac{\partial_{\alpha}}{H_{\alpha_{k}}} & 0 & 0 \\
0 & \frac{\partial_{\beta}}{H_{\beta_{k}}} & 0 \\
\frac{\partial_{\beta}}{H_{\beta_{k}}} & \frac{\partial_{\alpha}}{H_{\alpha_{k}}} & 0
\end{array}\right] ; \quad\left(A_{p}\right)_{i j}=\left[\begin{array}{ccc}
0 & 0 & \frac{1}{H_{\alpha_{k}} R_{\alpha_{k}}} \\
0 & 0 & \frac{1}{H_{\beta_{k}} R_{\beta_{k}}} \\
0 & 0 & 0
\end{array}\right]
$$

Analogously, the operators in Eq. (5b) are

$$
\begin{aligned}
&\left(D_{n \Omega}\right)_{i j}=\left[\begin{array}{ccc}
0 & 0 & \frac{\partial_{\alpha}}{H_{\alpha_{k}}} \\
0 & 0 & \frac{\partial_{\beta}}{H_{\beta_{k}}} \\
0 & 0 & 0
\end{array}\right] ; \quad\left(A_{n}\right)_{i j}=\left[\begin{array}{ccc}
\frac{-\lambda_{\mathrm{D}}}{H_{\alpha_{k}} R_{\alpha_{k}}} & 0 & 0 \\
0 & \frac{-\lambda_{\mathrm{D}}}{H_{\beta_{k}} R_{\beta_{k}}} & 0 \\
0 & 0 & 0
\end{array}\right] ; \\
&\left(D_{n z}\right)_{i j}=\left[\begin{array}{ccc}
\partial_{z} & 0 & 0 \\
0 & \partial_{z} & 0 \\
0 & 0 & \partial_{z}
\end{array}\right]
\end{aligned}
$$

The symbol $\partial_{\xi}$ stands for the partial differential operator with respect to the coordinate $\xi$, i.e. $\partial_{\xi}=\frac{\partial}{\partial \xi}$. The separation of terms and operators acting on the reference surface from those related to the thickness direction will result useful for the axiomatic modeling technique presented in Section 4.

The above geometrical relations retain all curvature terms. Simplified relations have been often proposed for thin (Love-type assumptions) and for shallow shells (Donnell-type assumptions). Within the thin shell assumption, terms of the type $z / R$ are neglected in Eq. (3), leading to the position $H_{\alpha_{k}}=H_{\beta_{k}}=1 . \lambda_{\mathrm{D}}$ is a trace operator allowing for the introduction of Donnell-type assumptions for shallow shells. Within this approximation the contributions of the in-plane (stretching) displacements $u_{\alpha}, u_{\beta}$ to the change of curvature are neglected.

The geometrical relations defining the electric field as a function of the electric potential read in curvilinear coordinates for the $k$ th layer

$$
\mathscr{E}_{i}^{k}=\left[\mathscr{E}_{\alpha_{k}} ; \mathscr{E}_{\beta_{k}} ; \mathscr{E}_{z_{k}}\right]^{\mathrm{T}}=\left[\begin{array}{lll}
-\frac{1}{H_{\alpha_{k}}} \frac{\partial \Phi^{k}}{\partial \alpha_{k}} & -\frac{1}{H_{\beta_{k}}} \frac{\partial \Phi^{k}}{\partial \beta_{k}} & -\frac{\partial \phi^{k}}{\partial z_{k}}
\end{array}\right]^{\mathrm{T}}
$$

where $\mathscr{E}_{i}^{k}$ is the electric field vector and $\Phi^{k}$ the electric potential. In order to handle matrices of equal dimensions, analogous to the ones quoted in Eq. (5), the above relation is exploded to a $3 \times 3$ system by writing the scalar potential $\Phi^{k}$ in a vector whose components are all identical, i.e. $\Phi_{i}^{k}=\left[\Phi^{k} ; \Phi^{k} ; \Phi^{k}\right]^{\mathrm{T}}$. Furthermore, the terms related to the in-plane and transverse behavior may be split leading finally to the following geometric relation defining the electric field:

$\mathscr{E}_{i}^{k}=\left(D_{\Omega e}\right)_{i j} \Phi_{j}^{k}+\left(D_{n e}\right)_{i j} \Phi_{j}^{k}$

with

$$
\left(D_{\Omega e}\right)_{i j}=\left[\begin{array}{ccc}
-\frac{\partial_{\alpha}}{H_{\alpha_{k}}} & 0 & 0 \\
0 & -\frac{\partial_{\beta}}{H_{\beta_{k}}} & 0 \\
0 & 0 & 0
\end{array}\right] ; \quad\left(D_{n e}\right)_{i j}=\left[\begin{array}{ccc}
0 & 0 & 0 \\
0 & 0 & 0 \\
0 & 0 & -\partial_{z}
\end{array}\right]
$$

\subsection{Constitutive equations}

In this work, a linearly coupled electro-mechanical material behavior is considered. Since we are concerned with a generalized displacement-based approach, the state function defining conveniently the energetic content of the material is the electric enthalpy $H$ (see, e.g., [25]). In vector notation, this state function reads 
$H=H\left(\epsilon_{r}, \mathscr{E}_{j}\right)=\frac{1}{2} \widetilde{C}_{q r}^{E} \epsilon_{q} \epsilon_{r}-e_{i q} \mathscr{E}_{i} \epsilon_{q}-\frac{1}{2} \varepsilon_{i j}^{S} \mathscr{E}_{i} \mathscr{E}_{j}$

In Eq. (10) $\widetilde{C}_{q r}^{E}$ is the matrix of the elastic moduli, $\varepsilon_{i j}^{S}$ is the matrix of the dielectric coefficients and $e_{i q}$ is the matrix containing the piezoelectric coefficients. The superscripts $E$ and $S$ of the matrices of the elastic and dielectric constants indicate that the coefficients are taken at constant electric field and at constant strain, respectively. The indices $q$ and $r$ run from 1 to 6 and define the 6 components of the engineering strain and stress vectors. Since the electric field is defined by only three components, the indices $i$ and $j$ range from 1 to 3 .

The constitutive equations are obtained upon derivation of the state function with respect to the independent variables:

$\sigma_{q}=\frac{\partial H}{\partial \epsilon_{q}}=\widetilde{C}_{q r}^{E} \epsilon_{r}-e_{q j} \mathscr{E}_{j}$

$\mathscr{D}_{i}=-\frac{\partial H}{\partial \mathscr{E}_{i}}=e_{i r} \epsilon_{r}+\varepsilon_{i j}^{S} \mathscr{E}_{j}$

Upon subdividing the in-plane (index $p$ ) and the transverse (index $n$ ) terms of the mechanical stresses and strains, the constitutive equations for each layer read

$\left(\sigma_{p}\right)_{i}^{k}=\left(\widetilde{C}_{p p}^{E}\right)_{i j}^{k}\left(\epsilon_{p}\right)_{j}^{k}+\left(\widetilde{C}_{p n}^{E}\right)_{i j}^{k}\left(\epsilon_{n}\right)_{j}^{k}-\left(e_{p}\right)_{i j}^{k} \mathscr{E}_{j}^{k}$

$\left(\sigma_{n}\right)_{i}^{k}=\left(\widetilde{C}_{n p}^{E}\right)_{i j}^{k}\left(\epsilon_{p}\right)_{j}^{k}+\left(\widetilde{C}_{n n}^{E}\right)_{i j}^{k}\left(\epsilon_{n}\right)_{j}^{k}-\left(e_{n}\right)_{i j}^{k} \mathscr{E}_{j}^{k}$

$\mathscr{D}_{i}^{k}=\left(e_{p}\right)_{i j}^{k}\left(\epsilon_{p}\right)_{j}^{k}+\left(e_{n}\right)_{i j}^{k}\left(\epsilon_{n}\right)_{j}^{k}+\left(\varepsilon^{S}\right)_{i j}^{k} \mathscr{E}_{j}^{k}$

where the following arrays characterizing a monoclinic material have been considered:

$$
\begin{aligned}
& \left(\widetilde{C}_{p p}^{E}\right)_{i j}^{k}=\left[\begin{array}{lll}
\widetilde{C}_{11} & \widetilde{C}_{12} & \widetilde{C}_{16} \\
\widetilde{C}_{12} & \widetilde{C}_{22} & \widetilde{C}_{26} \\
\widetilde{C}_{16} & \widetilde{C}_{26} & \widetilde{C}_{66}
\end{array}\right] ; \quad\left(\widetilde{C}_{p n}^{E}\right)_{i j}^{k}=\left[\begin{array}{ccc}
0 & 0 & \widetilde{C}_{13} \\
0 & 0 & \widetilde{C}_{23} \\
0 & 0 & \widetilde{C}_{36}
\end{array}\right] \\
& \left(\widetilde{C}_{n p}^{E}\right)_{i j}^{k}=\left[\begin{array}{ccc}
0 & 0 & 0 \\
0 & 0 & 0 \\
\widetilde{C}_{13} & \widetilde{C}_{23} & \widetilde{C}_{36}
\end{array}\right] ; \quad\left(\widetilde{C}_{n n}^{E}\right)_{i j}^{k}=\left[\begin{array}{ccc}
\widetilde{C}_{44} & \widetilde{C}_{45} & 0 \\
\widetilde{C}_{45} & \widetilde{C}_{55} & 0 \\
0 & 0 & \widetilde{C}_{33}
\end{array}\right]
\end{aligned}
$$

The dielectric and piezoelectric coefficients are written as

$$
\left(\varepsilon^{S}\right)_{i j}^{k}=\left[\begin{array}{ccc}
\varepsilon_{11} & 0 & 0 \\
0 & \varepsilon_{22} & 0 \\
0 & 0 & \varepsilon_{33}
\end{array}\right] ; \quad e_{i q}^{k}=\left[\begin{array}{cccccc}
0 & 0 & 0 & e_{14} & e_{15} & 0 \\
0 & 0 & 0 & e_{24} & e_{25} & 0 \\
e_{31} & e_{32} & e_{33} & 0 & 0 & e_{36}
\end{array}\right]
$$

The above matrix $e_{i q}^{k}$ is referred to a polarization axis aligned in the thickness direction, and it has been split in the two contributions (see Eq. (12)):

$$
\left(e_{p}\right)_{i j}^{k}=\left[\begin{array}{ccc}
0 & 0 & 0 \\
0 & 0 & 0 \\
e_{31} & e_{32} & e_{36}
\end{array}\right] ; \quad\left(e_{n}\right)_{i j}^{k}=\left[\begin{array}{ccc}
e_{14} & e_{15} & 0 \\
e_{24} & e_{25} & 0 \\
0 & 0 & e_{33}
\end{array}\right]
$$

The subscripts are related to the components in the curvilinear system according to the contracted notation $1=\alpha \alpha, 2=\beta \beta, 3=z z, 4=\beta z, 5=\alpha z, 6=\alpha \beta$.

Since this work deals with a closed-form solution technique, some additional symmetry properties for the constitutive law are enforced in order to avoid undesired couplings preventing an analytical solution. On the other hand, many practically implemented materials possess these higher-order symmetries. We thus limit the analysis to orthotropic materials, for which

$\widetilde{C}_{16}=\widetilde{C}_{26}=\widetilde{C}_{36}=\widetilde{C}_{45}=0$

must be set in the arrays Eq. (13). Concerning the piezoelectric layers, transversely isotropic materials with respect to the polarization axis are considered, and the following identities hold:

$e_{14}=e_{25}=e_{36}=0 ; \quad e_{31}=e_{32} ; \quad e_{15}=e_{24} ; \quad \varepsilon_{11}^{S}=\varepsilon_{22}^{S}$

\section{Thickness assumptions: the unified formulation}

The key step in any axiomatic modeling of plates and shells consists in the choice of the expansion of the unknowns in the thickness direction in order to reduce the problem to a two-dimensional one. In this work, the "Unified Formulation" according to Carrera [19] is employed. Within this formulation, an expansion is done for both the mechanical and electrical unknowns (displacements and electric potential, respectively) in each layer along the thickness coordinate $z_{k}$ according to the general form

$u_{i}^{k}\left(x, y, z_{k}\right)=f_{i}(x, y) F_{\tau}^{k}\left(z_{k}\right)\left(U_{i}^{k}\right)_{\tau}$
$\Phi^{k}\left(x, y, z_{k}\right)=g(x, y) F_{\tau}^{k}\left(z_{k}\right)\left(\Phi_{i}^{k}\right)_{\tau}$

with the summation index $\tau$ running from 1 to $N . N$ is the employed order of the expansion and is a free parameter of the formulation. By the extensive use of this index notation, a large variety of formulations for the multilayered structures can be derived by assembling differently the same basic arrays, called fundamental nuclei. This unique feature simplifies dramatically the implementation and comparison of many different theories.

The functions $F_{\tau}\left(z_{k}\right)$ are selected in different forms depending on the description method used for the model. In the case of Equivalent Single Layer (ESL) descriptions, a Taylor expansion is used according to

$F_{\tau}^{k}\left(z_{k}\right)=\left(z_{k}\right)^{\tau} \quad$ with $\tau=0,1, \ldots, N-1$

In this case, the unknowns related to $\tau=0$ are simply the values on the reference surface; the displacement unknowns associated with $\tau=1$ represent the rotations of the crosssection in an analogous way compared to the well-known First-Order Shear Deformation Theories (FSDT).

A refinement of ESL formulations may be achieved by superimposing to the above expansion the so-called 


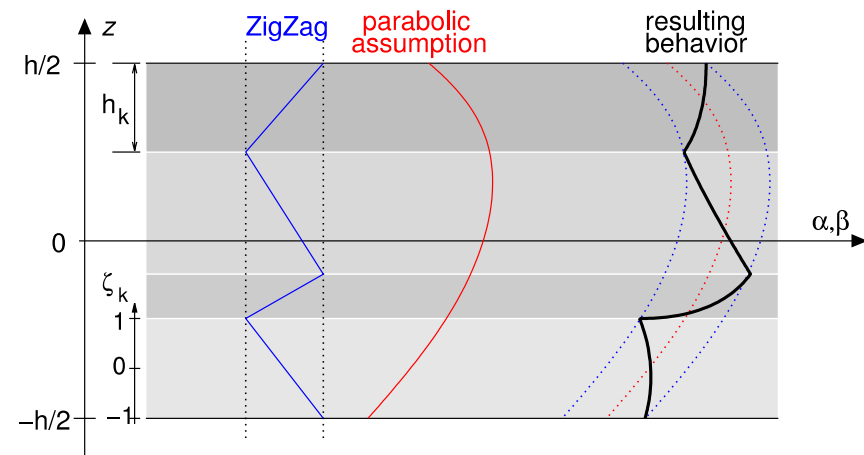

Fig. 2. Effects of MZZF in conjunction with a second-order ESL description.

Murakami ZigZag Function (MZZF), see also [26,27]. This function allows the introduction of a discontinuity in the slopes of the displacements and thus represents a step towards the fulfillment of the $C_{z}^{0}$-requirements. In this latter case, the expansion Eq. (19) is extended by the ZigZag term yielding for the general unknown $\mathscr{U}$ the following through-thickness approach

$\mathscr{U}\left(z_{k}\right)=\left(z_{k}\right)^{\tau} \mathscr{U}_{\tau}+(-1)^{k} \zeta_{k} \mathscr{U}_{Z Z} \quad$ with $\zeta_{k}=\frac{2 z_{k}}{h_{k}}$

where $h_{k}$ is the thickness of the layer. Observing Fig. 2, it is important to underline that the MZZF introduces a dependency of the through-thickness behavior on the layer number within a layer-independent ESL formulation. Nonetheless, the added unknown $\mathscr{U}_{Z Z}$ assumes the same value for each layer, thus respecting the layer-independency of the basis formulation.

Layerwise (LW) descriptions require in the assembly procedure to respect the interlaminar continuity of the displacements and of the electric potential: for this purpose, Legendre polynomials $P\left(\zeta_{k}\right)$ are suitable for building the interpolating functions $F_{\tau}\left(z_{k}\right)$ [28]

$F_{t}^{k}\left(z_{k}\right)=\frac{P_{0}\left(\zeta_{k}\right)+P_{1}\left(\zeta_{k}\right)}{2} ; \quad F_{b}^{k}\left(z_{k}\right)=\frac{P_{0}\left(\zeta_{k}\right)-P_{1}\left(\zeta_{k}\right)}{2}$

$F_{\tau}^{k}\left(z_{k}\right)=P_{\tau}-P_{\tau-2} \quad$ with $\tau=2,3, \ldots, N$

In this case, the unknowns associated to the linear expansion for $N=1$ represent the values of the unknowns at the top $\left(F_{t}\right)$ and bottom $\left(F_{b}\right)$ of the layer. Additional nodes along the $z_{k}$ axis allow then a higher-order distribution of the unknowns in the layer thickness. Interlaminar Continuity (IC) is hereafter enforced by simply setting

$\mathscr{U}_{\tau=t}^{k}=\mathscr{U}_{\tau=b}^{k+1}$

\subsection{Employed assumptions for the primary variables}

The primary variables for the present generalized displacement-based approach are the mechanical displacements $u_{i}$ and the electric potential $\Phi$. In the following, for the displacements either ESL or LW descriptions are used, while only a LW description of the electric potential is con- sidered. This choice seems reasonable since it may well capture the steep local gradients arising from the sharp discontinuities of the electrical properties between piezoelectric and passive layers.

Furthermore, without loss of generality, we restrict our analysis to formulations with the same order of expansion $N$ for all involved fields, irrespective of the description method selected for the displacements. Formulations based on an ESL description are denoted as EDN models; if the MZZF is superimposed to the ED-formulation, the model will be indicated as EDZN. Models based on a layerwise description of the displacements will be denoted by the acronym $\mathrm{LD} N$.

Linear $(N=1)$ up to fourth-order $(N=4)$ expansions in the thickness direction are considered. In total, 11 different formulations are derived and compared. Additionally, simplified models like FSDT or Classical Lamination Theory (CLT) may be recovered enforcing some transverse contributions associated to the ED1 model to vanish: this is implemented within a typical penalty technique.

The proposed model will be used in the next section to derive the governing equations for the free-vibration problem of multilayered shells with embedded piezoelectric layers.

\section{Governing equations}

The equations are presented within a variational formulation in the framework of Hamilton's principle. Due to the very different time-scales of the involved electrical and mechanical field the former one can be considered as quasi-static: electrical inertia effects are therefore neglected. The variational statement is conveniently formulated in the standard tensorial notation $[7,9]$

$$
\begin{aligned}
& \delta\left[\int_{t_{0}}^{t_{1}} \mathrm{~d} t \int_{V}-\frac{1}{2} \rho \dot{u}_{i} \dot{u}_{i}+H\left(\epsilon_{q r}, \mathscr{E}_{i}\right) \mathrm{d} V\right] \\
& \quad-\delta\left[\int_{t_{0}}^{t_{1}} \mathrm{~d} t \int_{\Gamma} u_{i} \bar{t}_{i}-\Phi \bar{q}-t_{i}\left(u_{i}-\bar{u}_{i}\right)-q(\Phi-\bar{\Phi}) \mathrm{d} S\right]=0
\end{aligned}
$$

$t_{i}$ represents the surface tractions and $q$ the surface charge densities; quantities with a bar are prescribed on the domain boundary $\Gamma$. For a multilayered shell, Eq. (23) can be re-written for each layer by including additional boundary terms related to the interlaminar surfaces of each layer. However, these additional boundary terms vanish in the multilayered body in the assembly procedure by canceling each other, provided the layers are perfectly bonded and with a unique electric potential. Hence, without considering these terms and splitting the in-plane and transverse strain energies according to Eq. (5), an equivalent form for Eq. (23) is

$$
\begin{aligned}
& \int_{t_{0}}^{t_{1}} \mathrm{~d} t \sum_{k=1}^{N_{l}} \int_{V_{k}}\left(\delta \epsilon_{p}\right)_{i}^{k}\left(\sigma_{p}\right)_{i}^{k}+\left(\delta \epsilon_{n}\right)_{i}^{k}\left(\sigma_{n}\right)_{i}^{k}-\delta \mathscr{\mathscr { C }}_{i}^{k} \mathscr{D}_{i}^{k} \\
& \quad+\delta u_{i}^{k} \rho^{k} \ddot{u}_{i}^{k} \mathrm{~d} V=0
\end{aligned}
$$


Note that, according to the electric enthalpy formulation, the virtual variations have been applied to the displacements and the electric potential. Introduction of both the constitutive equations Eq. (12) and the geometric relations Eqs. $(5,9)$ yields the following variational equation:

$$
\begin{aligned}
& \int_{t_{0}}^{t_{1}} \mathrm{~d} t \sum_{k=1}^{N_{l}} \int_{V_{k}}\left[\left(\left(D_{p}\right)_{l i}+\left(A_{p}\right)_{l i}\right) \delta u_{i}^{k}\right]^{\mathrm{T}}\left(\widetilde{C}_{p p}^{E}\right)_{l m}^{k}\left[\left(D_{p}\right)_{m j}+\left(A_{p}\right)_{m j}\right] u_{j}^{k} \\
& +\left[\left(\left(D_{p}\right)_{l i}+\left(A_{p}\right)_{l i}\right) \delta u_{i}^{k}\right]^{\mathrm{T}}\left(\widetilde{C}_{p n}^{E}\right)_{l m}^{k}\left[\left(D_{n \Omega}\right)_{m j}+\left(A_{n}\right)_{m j}+\left(D_{n z}\right)_{m j}\right] u_{j}^{k} \\
& +\left[\left(\left(D_{n \Omega}\right)_{l i}+\left(A_{n}\right)_{l i}+\left(D_{n z}\right)_{l i}\right) \delta u_{i}^{k}\right]^{\mathrm{T}}\left(\widetilde{C}_{n p}^{E}\right)_{l m}^{k}\left[\left(D_{p}\right)_{m j}+\left(A_{p}\right)_{m j}\right] u_{j}^{k} \\
& +\left[\left(\left(D_{n \Omega}\right)_{l i}+\left(A_{n}\right)_{l i}+\left(D_{n z}\right)_{l i}\right) \delta u_{i}^{k}\right]^{\mathrm{T}}\left(\widetilde{C}_{n n}^{E}\right)_{l m}^{k}\left[\left(D_{n \Omega}\right)_{m j}+\left(A_{n}\right)_{m j}\right. \\
& \left.+\left(D_{n z}\right)_{m j}\right] u_{j}^{k}-\left[\left(\left(D_{p}\right)_{l i}+\left(A_{p}\right)_{l i}\right) \delta u_{i}^{k}\right]^{\mathrm{T}}\left(e_{p}\right)_{l m}^{k}\left(\left(D_{\Omega e}\right)_{m j}+\left(D_{n e}\right)_{m j}\right) \Phi_{j}^{k} \\
& -\left[\left(\left(D_{n \Omega}\right)_{l i}+\left(A_{n}\right)_{l i}+\left(D_{n z}\right)_{l i}\right) \delta u_{i}^{k}\right]^{\mathrm{T}}\left(e_{n}\right)_{l m}^{k}\left(\left(D_{\Omega e}\right)_{m j}+\left(D_{n e}\right)_{m j}\right) \Phi_{j}^{k} \\
& -\left[\left(\left(D_{\Omega e}\right)_{l i}+\left(D_{n e}\right)_{l i}\right) \delta \Phi_{i}^{k}\right]^{\mathrm{T}}\left(\left(e_{p}\right)_{l m}^{k}\left[\left(D_{p}\right)_{m j}+\left(A_{p}\right)_{m j}\right]\right. \\
& \left.+\left(e_{n}\right)_{l m}^{k}\left[\left(D_{n \Omega}\right)_{m j}+\left(A_{n}\right)_{m j}+\left(D_{n z}\right)_{m j}\right]\right) u_{j}^{k}-\left[\left(\left(D_{\Omega e}\right)_{l i}+\left(D_{n e}\right)_{l i}\right) \delta \Phi_{i}^{k}\right]^{\mathrm{T}} \\
& \times\left(\varepsilon^{S}\right)_{l m}^{k}\left(\left(D_{\Omega e}\right)_{m j}+\left(D_{n e}\right)_{m j}\right) \Phi_{j}^{k}+\delta u_{i}^{k} \rho^{k} \ddot{u}_{i}^{k} \mathrm{~d} V=0
\end{aligned}
$$

Upon introduction of the assumptions $F_{s}$ for the throughthickness behavior of the unknowns and $F_{\tau}$ for the weighting functions according to Eq. (18b), the domain integral is conveniently split into the integration in thickness direction $z_{k}$ and the integration on the reference surface $\Omega_{k}$. Considering the integration on the reference surface, in order to shift the differential operators acting on the in-plane coordinates from the weighting functions to the unknowns, an integration by parts according to the scheme

$$
\begin{aligned}
\int_{\Omega_{k}}\left(\left(D_{\Omega}\right)_{j i} \delta u_{i}^{k}\right)^{\mathrm{T}} u_{j}^{k} \mathrm{~d} \Omega= & -\int_{\Omega_{k}} \delta u_{i}^{k^{\mathrm{T}}}\left(\left(D_{\Omega}\right)_{i j} u_{j}^{k}\right) \mathrm{d} \Omega \\
& +\int_{\Gamma_{k}} \delta u_{i}^{k^{\mathrm{T}}}\left(\left(I_{\Omega}\right)_{i j} u_{j}^{k}\right) \mathrm{d} \Gamma
\end{aligned}
$$

is done. The operators interested by this transformations are $\left(D_{p}\right)_{i j},\left(D_{n \Omega}\right)_{i j}$ and $\left(D_{\Omega e}\right)_{i j}$. The matrices resulting in the boundary terms are

$$
\begin{aligned}
& \left(I_{p}\right)_{i j}=\left[\begin{array}{ccc}
\frac{1}{H_{\alpha_{k}}} & 0 & 0 \\
0 & \frac{1}{H_{\beta_{k}}} & 0 \\
\frac{1}{H_{\beta_{k}}} & \frac{1}{H_{\alpha_{k}}} & 0
\end{array}\right] ; \quad\left(I_{n \Omega}\right)_{i j}=\left[\begin{array}{ccc}
0 & 0 & \frac{1}{H_{\alpha_{k}}} \\
0 & 0 & \frac{1}{H_{\beta_{k}}} \\
0 & 0 & 0
\end{array}\right] ; \\
& \left(I_{\Omega e}\right)_{i j}=\left[\begin{array}{ccc}
-\frac{1}{H_{\alpha_{k}}} & 0 & 0 \\
0 & -\frac{1}{H_{\beta_{k}}} & 0 \\
0 & 0 & 0
\end{array}\right]
\end{aligned}
$$

The integrations in thickness direction may then be performed in a direct manner: this allows a straightforward and exact evaluation of all curvature contributions, involving terms multiplied or divided by the metric coefficients $H_{\alpha_{k}}$ and $H_{\beta_{k}}$. These integrals are quoted in Appendix A.

Finally, since the virtual variations of the displacements $\delta u_{i \tau}^{k}$ and of the electric potential $\delta \Phi_{i \tau}^{k}$ are independent, the governing equations for the resulting coupled electromechanical system can be written in the following set of equations:

$\delta u_{i \tau}^{k}:\left(K_{u u}^{\tau s k}\right)_{i j} u_{j s}^{k}+\left(K_{u e}^{\tau s k}\right)_{i j} \Phi_{j s}^{k}=-\left(M^{\tau s k}\right)_{i j} \ddot{u}_{j s}^{k}+\left(p_{m \tau}\right)_{i}$

$\delta \Phi_{i \tau}^{k}: \quad\left(K_{e u}^{\tau s k}\right)_{i j} u_{j s}^{k}+\left(K_{e e}^{\tau s k}\right)_{i j} \Phi_{j s}^{k}=\left(p_{e \tau}\right)_{i}$

The arrays $\left(p_{m \tau}\right)_{i}$ and $\left(p_{e \tau}\right)_{i}$ indicate the variationally consistent mechanical and electrical loads, respectively. Along with these governing equations the following boundary conditions hold:

$u_{i \tau}^{k}=\bar{u}_{i \tau}^{k} \quad$ or

$\left(\Pi_{u u}^{\tau s k}\right)_{i j} u_{j s}^{k}+\left(\Pi_{u e}^{\tau s k}\right)_{i j} \Phi_{j s}^{k}=\left(\Pi_{u u}^{\tau s k}\right)_{i j} \bar{u}_{j s}^{k}+\left(\Pi_{u e}^{\tau s k}\right)_{i j} \bar{\Phi}_{j s}^{k}$

$\Phi_{i \tau}^{k}=\bar{\Phi}_{i \tau}^{k} \quad$ or

$\left(\Pi_{e u}^{\tau s k}\right)_{i j} u_{j s}^{k}+\left(\Pi_{e e}^{\tau s k}\right)_{i j} \Phi_{j s}^{k}=\left(\Pi_{e u}^{\tau s k}\right)_{i j} \bar{u}_{j s}^{k}+\left(\Pi_{e e}^{\tau s k}\right)_{i j} \bar{\Phi}_{j s}^{k}$

The matrices $K_{i j}, M_{i j}$ and $\Pi_{i j}$ are the so called fundamental nuclei of the formulation: these represent the stiffness contributions for each layer and for each couple $\tau, s$ of the selected order of the expansion. These expressions are invariant with respect to both the different description level (ESL or LW) and the expansion order $N$ for the throughthickness assumptions. The fundamental nuclei are quoted in Appendix A. The assembly procedures to build the arrays for the multilayered structure are described in the next section.

\subsection{Assembly of system arrays}

The contributions of the single terms $\tau, s$ are first assembled at layer level following the scheme depicted in Fig. 3. For an LW description, the layer-specific arrays are assembled accounting for the continuity of the degrees of freedom (DOFs) at the interface according to Eq. (22). ESL descriptions require a simple superimposition of the layer-specific contributions since the layer-specific DOFs are coincident with the DOFs of the multilayered structure. For clarity, the two different assembly procedures for the two description methods are depicted in Fig. 4.

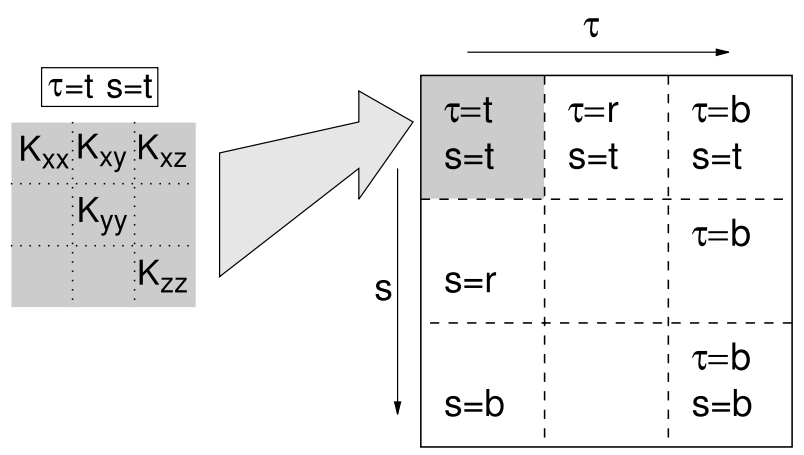

Fig. 3. Expansion of the fundamental nuclei to the layer-specific contributions. 


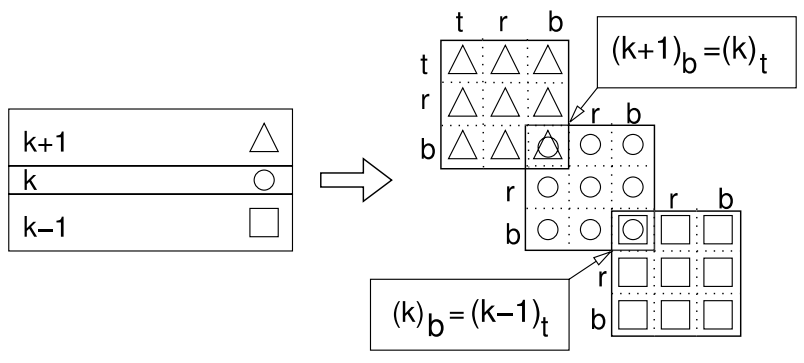

(a) Multilayer assembly for LW descriptions

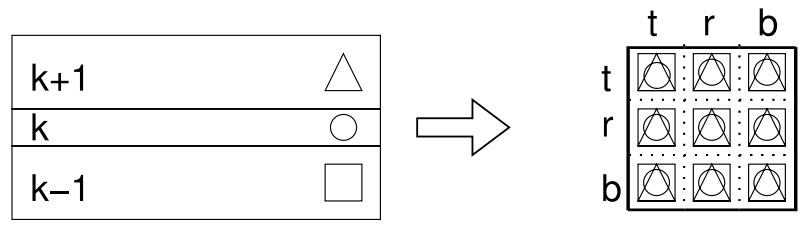

(b) Multilayer assembly for ESL descriptions

Fig. 4. Schemes describing the assembly procedures of multilayered contributions.

\subsection{Closed-form solution for the free-vibration problem}

For the derived boundary value problem, for particular geometry, material symmetry and boundary conditions, an analytical solution can be derived. For simply supported shells, a Navier-type closed-form solution may be found with the following expressions:

$u_{\alpha \tau}^{k}\left(\alpha_{k}, \beta_{k} ; t\right)=\hat{u}_{\alpha \tau}^{k} \cos \left(\frac{m \pi \alpha_{k}}{a_{k}}\right) \sin \left(\frac{n \pi \beta_{k}}{b_{k}}\right) \mathrm{e}^{\mathrm{i} \omega_{m n} t}$

$u_{\beta \tau}^{k}\left(\alpha_{k}, \beta_{k} ; t\right)=\hat{u}_{\beta \tau}^{k} \sin \left(\frac{m \pi \alpha_{k}}{a_{k}}\right) \cos \left(\frac{n \pi \beta_{k}}{b_{k}}\right) \mathrm{e}^{\mathrm{i} \omega_{m n} t}$

$u_{z \tau}^{k}\left(\alpha_{k}, \beta_{k} ; t\right)=\hat{u}_{z \tau}^{k} \sin \left(\frac{m \pi \alpha_{k}}{a_{k}}\right) \sin \left(\frac{n \pi \beta_{k}}{b_{k}}\right) \mathrm{e}^{\mathrm{i} \omega_{m n} t}$

$\Phi_{\tau}^{k}\left(\alpha_{k}, \beta_{k} ; t\right)=\widehat{\Phi}_{\tau}^{k} \sin \left(\frac{m \pi \alpha_{k}}{a_{k}}\right) \sin \left(\frac{n \pi \beta_{k}}{b_{k}}\right) \mathrm{e}^{\mathrm{i} \omega_{m n} t}$

$a_{k}$ and $b_{k}$ are the arc lengths of the shell along the two coordinates $\alpha$ and $\beta . m$ and $n$ represent the number of halfwaves in $\alpha$ and $\beta$ direction, respectively. These numbers characterize the vibration mode associated to the circular frequency $\omega_{m n} . \mathrm{i}=\sqrt{-1}$ is the imaginary unit and $t$ the time.

In the remainder of this article, only the free-vibration analysis will be addressed. The external loading (mechanical and electrical) is therefore set to zero. Introduction of the expressions Eq. (30) into the governing equations (28) leads to a linear set of ordinary differential equations in time:

$\left(\widehat{K}_{u u}\right)_{i j} \hat{u}_{j}+\left(\widehat{K}_{u e}\right)_{i j} \widehat{\Phi}_{j}=\omega_{m n}^{2}(\hat{M})_{i j} \hat{u}_{j}$

$\left(\widehat{K}_{e u}\right)_{i j} \hat{u}_{j}+\left(\widehat{K}_{e e}\right)_{i j} \widehat{\Phi}_{j}=0$
A hat denotes quantities obtained after introduction of the harmonic functions into the governing equations. The coupled electro-mechanical system can be conveniently reduced to a purely mechanical one by condensing statically the electrical unknowns:

$\widehat{\Phi}_{j}=-\left(\widehat{K}_{e e}\right)_{i j}^{-1}\left(\widehat{K}_{e u}\right)_{i j} \hat{u}_{j}$

Substitution of this relation into the mechanical equation gives

$$
\left[\left(\widehat{K}_{u u}\right)_{i j}-\left(\widehat{K}_{u e}\right)_{i j}\left(\widehat{K}_{e e}\right)_{i j}^{-1}\left(\widehat{K}_{e u}\right)_{i j}\right] \hat{u}_{j}=\omega_{m n}^{2}(\hat{M})_{i j} \hat{u}_{j}
$$

As usual, the free-vibration response of the condensed system Eq. (33) is computed as solution of the associated eigenvalue problem

$\left\|K_{i j}-\lambda_{m n} M_{i j}\right\|=0$

where $\lambda_{m n}=\omega_{m n}^{2}$ and

$K_{i j}=\left(\widehat{K}_{u u}\right)_{i j}-\left(\widehat{K}_{u e}\right)_{i j}\left(\widehat{K}_{e e}\right)_{i j}^{-1}\left(\widehat{K}_{e u}\right)_{i j}$

The eigenvectors $\hat{u}_{i}^{(m n)}$ associated to the eigenvalues $\lambda_{m n}$ define the vibration modes in terms of the mechanical displacements. The electrical modes are recovered from the mechanical ones according to Eq. (32) as

$\widehat{\Phi}_{i}^{(m n)}=-\left(\widehat{K}_{e e}\right)_{i j}^{-1}\left(\widehat{K}_{e u}\right)_{i j} \hat{u}_{j}^{(m n)}$

\section{Free-vibration analysis of piezoelectric shells}

Previous studies have shown that higher-order thickness expansions together with the closed-form analytical solution technique yield a simulation method, which is capable to recover the exact three-dimensional response of laminated elastic shells [29] and of laminated piezoelectric plates [22]. For the free-vibration problem of multilayered, piezoelectric shells only few works are available in open literature presenting exact 3D-solutions. Verification computations are presented considering the results obtained by Drumheller and Kalnins in [30] for an homogeneous piezoelectric cylinder, and those obtained by Heyliger et al. in [13] for a laminated ring. Finally, the eigenfrequencies of a laminated cylinder are presented and the effects of the electro-mechanical coupling are discussed.

\subsection{Numerical verification}

Drumheller and Kalnins analyze the free-vibration response of a simply supported cylinder constituted by homogeneous piezoelectric material with short-circuited surfaces [30]. With reference to Fig. 5, cylinders of variable length $a$ are considered; the mean line radius is $R_{\beta}=11.1125 \mathrm{~mm}$ and the shell thickness is $h=3.175 \mathrm{~mm}$ (the material data are reported in Table 1). The two considered vibration modes are schematically represented in Fig. 6. These modes are obtained in the present theory by setting $m=1$ (fundamental mode in the cylinder axis) and $n=0$ (axisymmetric mode, i.e. $\partial_{\beta}=0$ ). 


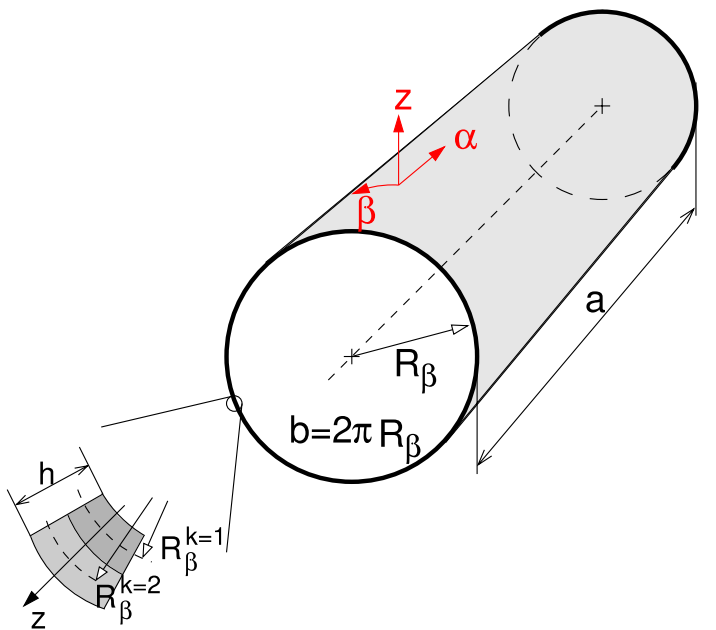

Fig. 5. Geometry and notation employed for the description of a layered, closed cylindrical shells $\left(R_{\alpha}=\infty\right)$. Case of a laminate with 2 layers.

Table 1

Material data for the piezoelectric cylinder [30]

\begin{tabular}{lrll}
\hline \multicolumn{2}{l}{ Material constants for PZT-4 } & & \\
$C_{11}^{E}=C_{22}^{E}$ & $139.89 \mathrm{GPa}$ & $e_{31}=e_{32}$ & $-5.5864 \mathrm{C} / \mathrm{m}^{2}$ \\
$C_{33}^{E}$ & $113.16 \mathrm{GPa}$ & $e_{33}$ & $16.3801 \mathrm{C} / \mathrm{m}^{2}$ \\
$C_{12}^{E}$ & $78.62 \mathrm{GPa}$ & $e_{15}=e_{24}$ & $12.2013 \mathrm{C} / \mathrm{m}^{2}$ \\
$C_{13}^{E}=C_{23}^{E}$ & $76.54 \mathrm{GPa}$ & $\varepsilon_{11}^{S}=\varepsilon_{22}^{S}$ & $59.77 \times 10^{-10} \mathrm{C} / \mathrm{V} \mathrm{m}$ \\
$C_{44}^{E}=C_{55}^{E}$ & $26.91 \mathrm{GPa}$ & $\varepsilon_{33}^{S}$ & $58.57 \times 10^{-10} \mathrm{C} / \mathrm{V} \mathrm{m}$ \\
$C_{66}^{E}$ & $30.64 \mathrm{GPa}$ & $\rho$ & $7.5 \times 10^{3} \mathrm{~kg} / \mathrm{m}^{3}$ \\
\hline
\end{tabular}

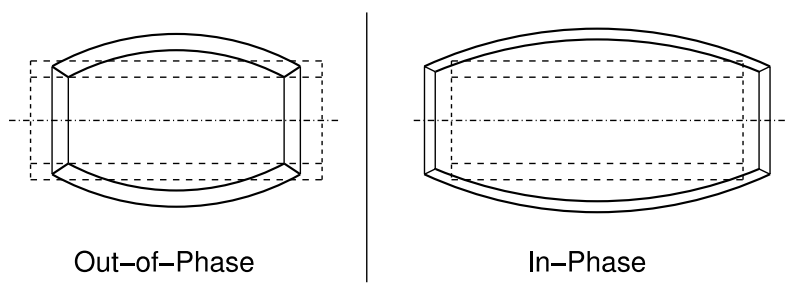

Fig. 6. Axisymmetric modes of a piezoelectric cylinder considered in [30].

The results are given in Fig. 7. For the out-of-phase (OP) mode, a very good agreement with the reference results can be recognized for both the models involving a linear and a fourth-order assumption for the thickness distributions. For the in-phase (IP) mode, the results of the ED1 and the ED4 formulations show a larger difference due to the more important role played by the thickness effects compared to the OP-mode. However, the reference solution is seen to lie in-between the closed-form solutions obtained for the most accurate ED4 formulation and the less accurate ED1 formulation. Note that the electric stiffness term, represented by $\left(\widehat{K}_{e e}\right)_{i j}$ in Eq. (31b), vanishes for a linear distribution of the electric potential. Recalling the approximations introduced in the reference solution [30], the results depicted in Fig. 7 confirm the validity of the proposed model. Finally, note that the numerical values of

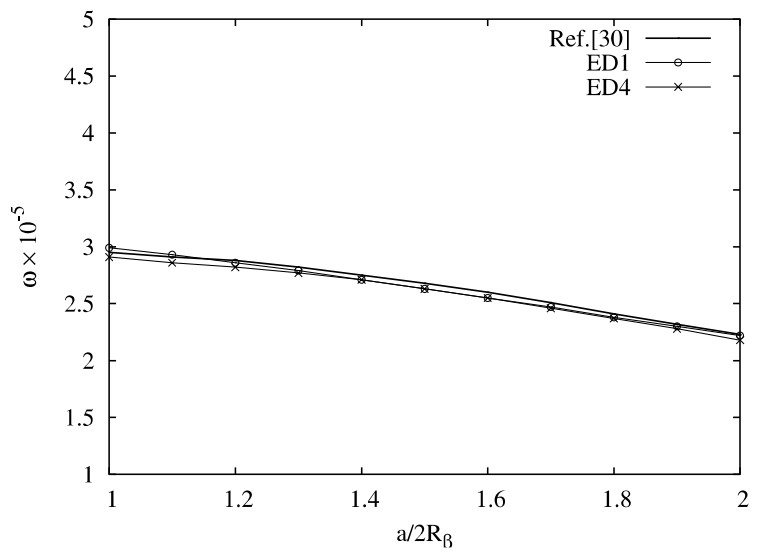

(a) Out-of-Phase Mode

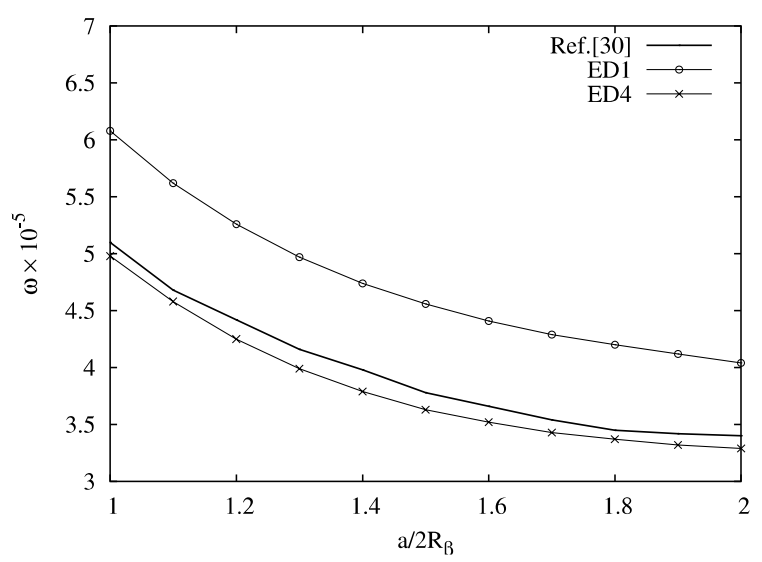

(b) In-Phase Mode

Fig. 7. Free-vibration frequencies $[\mathrm{rad} / \mathrm{s}]$ for the piezoelectric cylinder [30].

Drumheller and Kalnins have been graphically extracted from a picture included in the cited reference; therefore, only a qualitative assessment has been proposed in the present work.

Heyliger et al. calculate the natural frequencies of a laminated ring consisting of an inner isotropic, elastic layer of Titanium (Ti) and an outer piezoceramic layer (PZT-4) [13]. The geometry of the ring is depicted in Fig. 8 (left), where the coordinates and notations defined in Fig. 5 have been employed. To reduce the computational cost, only a quarter of the ring is considered in [13], and various symmetry conditions holding on the edges $\theta=0, \theta=\pi / 2$ and $x=0$ (see Fig. 8) are given as boundary conditions for the dynamic analysis. The piezoelectric layer is polarized in radial direction and has a thickness of $h_{P}=0.25 h=$ $0.001 \mathrm{~m}$. The employed material parameters for the titanium layer are $E=114 \mathrm{GPa}, v=0.3, \rho=2768 \mathrm{~kg} / \mathrm{m}^{3}$ and $\varepsilon=\varepsilon_{0}=8.85 \times 10^{-12} \mathrm{C} / \mathrm{Vm}$; those for PZT-4 are given in Fig. 8 (right).

In [13], a Finite Element (FE) formulation is presented based on a layerwise description of the coupled electromechanical problem. The symmetry conditions corresponding to a vanishing circumferential displacement on 


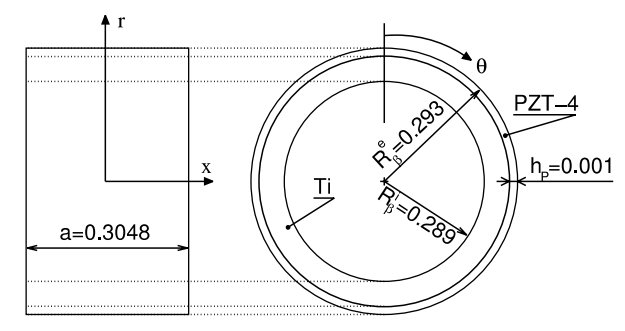

$$
\begin{array}{ll}
\hline E_{1}=81.3 \mathrm{GPa} & e_{15}=12.72 \mathrm{C} / \mathrm{m}^{2} \\
E_{3}=64.5 \mathrm{GPa} & e_{31}=-5.20 \mathrm{C} / \mathrm{m}^{2} \\
\nu_{13}=0.432 & e_{33}=15.08 \mathrm{C} / \mathrm{m}^{2} \\
\nu_{12}=0.329 & \varepsilon_{11}=1475 \varepsilon_{0} \\
G_{44}=25.6 \mathrm{GPa} & \varepsilon_{33}=1300 \varepsilon_{0} \\
\rho=7600 \mathrm{~kg} / \mathrm{m}^{3} & \varepsilon_{0}=8.8510^{-12} \mathrm{C} / \mathrm{Vm} \\
\hline
\end{array}
$$

Fig. 8. Left: Geometry of the laminated ring considered in [13] (all quantities given in [m]). Right: Data for transversally isotropic PZT-4 material (note: $\left.G_{12}=\frac{E_{1}}{2\left(1+v_{12}\right)}\right)$.

Table 2

Natural frequencies $[\mathrm{Hz}]$ for the layered ring

\begin{tabular}{llllll}
\hline Ref. [13] & $n=4$ & $n=8$ & $n=12$ & $n=16$ & $n=20$ \\
& 31.27 & 170.42 & 407.29 & 445.21 & 1190.48 \\
\hline LD4 & $31.64(1.18)$ & $171.60(0.69)$ & $406.87(0.10)$ & $736.08(1.23)$ & $1158.53(2.68)$ \\
LD3 & $31.64(1.18)$ & $171.60(0.69)$ & $406.87(0.10)$ & $736.08(1.23)$ & $1158.53(2.68)$ \\
LD2 & $31.64(1.18)$ & $171.60(0.69)$ & $406.89(0.09)$ & $736.12(1.22)$ & $1158.63(2.68)$ \\
LD1 & $33.28(6.43)$ & $180.51(5.92)$ & $427.99(5.08)$ & $774.25(3.90)$ & $1218.56(2.36)$ \\
ED4 & $31.65(1.2)$ & $171.64(0.72)$ & $406.98(0.08)$ & $736.28(1.20)$ & $1158.87(2.66)$ \\
ED3 & $31.65(1.22)$ & $171.65(0.72)$ & $407.00(0.07)$ & $736.32(1.19)$ & $1158.92(2.65)$ \\
ED2 & $31.66(1.25)$ & $171.70(0.75)$ & $407.13(0.04)$ & $736.59(1.16)$ & $1159.43(2.61)$ \\
ED1 & $35.54(13.66)$ & $192.74(13.10)$ & $456.95(12.19)$ & $826.54(10.91)$ & $1300.67(9.26)$ \\
\hline
\end{tabular}

The values in parentheses are the relative percentual differences with respect to the reference solution.

the edges $\theta=0$ and $\theta=\pi / 2$ as well as to a vanishing axial displacement at $x=0$ (group 7 of the cited reference) are represented within the present formulation by $m=0$ and $n=4,8,12,16,20$. Only the mode characterized by a pure circumferential deformation has been considered, i.e. $\hat{u}_{\alpha \tau}^{k}=0$. The electrical boundary conditions consist of a short-circuit between the top and bottom surface of the piezoelectric layer. Table 2 reports the results obtained by the present formulations as well as the relative difference (in percent) with respect to the values in [13]. It can be seen that a qualitative jump is associated to the consideration of the electrical stiffness introduced by an at least quadratic assumption for the electric potential: in fact, all linear formulations (LD1, ED1) provide results of clearly lower quality, the related models resulting always too stiff. Except for slight differences in the higher modes, the closed-form solutions of all formulations present a good agreement with the approximate FE solution proposed in literature. It is interesting to note that, for higher modes, the analytical solution for less accurate formulations represents better the FE solution. This may be due to the superimposition of two distinct errors, i.e. the discretization error and the error in the thickness assumptions, which cancel out, thus providing a better agreement.

\subsection{Electro-mechanical coupling in free-vibrations of adaptive laminates}

The aim is to systematically evaluate the influence of the electro-mechanical coupling on the free-vibration charac- teristics of multilayered plates and shells with embedded piezoelectric materials. In this work, the parameter indicating the amount of electro-mechanical interaction is selected to be the relative difference between the frequencies of the fully coupled system and the frequencies obtained neglecting the piezoelectric coefficients, i.e. setting $e_{i q}^{k}=0$. Thus, in the latter case the piezoelectric materials give only a mechanical contribution to the shell stiffness. Note that an alternative definition of the amount of electro-mechanical interaction in smart shells has been proposed by Rogacheva [14] and applied, e.g., by Benjeddou [31]: it considers the relative difference between the natural frequencies related to the piezo-layers working under open-circuit condition and the natural frequencies obtained with the piezo-layers working in short-circuit.

The simply supported, 5-layered square piezoelectric plate, for which Heyliger and Saravanos presented in [32] an exact 3D solution, is considered. Ballhause et al. could recover the exact 3D solution within the "Unified Formulation" [22]. The geometrical and material data as well as the electrical and mechanical boundary conditions can be taken from the cited references. The first frequency parameter $\gamma_{1}=\omega_{1} \times 10^{-2}$ of the plate is given in Table 3 for both the coupled electro-mechanical case (LD4) and the uncoupled case $(\mathrm{LD} 4 \mathrm{~m})$. Thin $(a / h=100)$ and thick $(a / h=4)$ plates are considered. For the considered geometries, an influence between $1.5 \%$ and $5.5 \%$ has been obtained. The piezo-layers are polarized in thickness direction and the contributions of the electrical stiffness to the resulting plate stiffness increase with the slenderness of the 
Table 3

Influence of piezoelectric coupling on the first frequency parameter $\gamma_{1}=\omega_{1} \times 10^{-2}[\mathrm{rad} / \mathrm{s}]$ for a simply supported, laminated square plate

\begin{tabular}{llrrrr}
\hline & \multicolumn{1}{l}{$l / h$} & & & \\
\cline { 2 - 6 } & 2 & 4 & 10 & 50 & 100 \\
\hline LD4 & 136604 & 57074.0 & 13526.4 & 618.104 & 155.285 \\
LD4m & 134551 & 55514.8 & 12905.6 & 584.085 & 146.680 \\
$\Delta[\%]$ & 1.50 & 2.73 & 4.59 & 5.50 & 5.54 \\
\hline
\end{tabular}

Geometry and material parameters given in [32].

Table 4

Effect of modeling on the piezoelectric coupling (first frequency parameter $\left.\gamma_{1}=\omega_{1} \times 10^{-2}\right)[\mathrm{rad} / \mathrm{s}]$

\begin{tabular}{|c|c|c|c|c|c|c|}
\hline & \multicolumn{3}{|c|}{$a / h=100$} & \multicolumn{3}{|l|}{$a / h=4$} \\
\hline & Coupled & Uncoupled & $\Delta[\%]$ & Coupled & Uncoupled & $\Delta[\%]$ \\
\hline LD1 & 155.508 & 146.953 & 5.50 & 57252.5 & 55754.8 & 2.61 \\
\hline ED4 & 155.319 & 146.825 & 5.47 & 58713.8 & 56939.3 & 3.02 \\
\hline EDZ3 & 155.329 & 147.021 & 5.35 & 57656.7 & 56185.2 & 2.15 \\
\hline ED2 & 155.450 & 147.124 & 5.36 & 69413.7 & 66641.7 & 3.99 \\
\hline EDZ1 & 172.847 & 171.773 & 0.62 & 63204.7 & 63030.6 & 0.28 \\
\hline ED1 & 172.956 & 171.884 & 0.62 & 74105.9 & 73720.9 & 0.52 \\
\hline
\end{tabular}

Geometry and material parameters given in [32].

plate. Note that a saturation effect arises for the thin plate limit, i.e. the rate of the increase of the influence with the slenderness tends to zero for very thin plates. These considerations are completed by an analysis of the effects of the model accuracy on the capability to capture the physics of the electro-mechanical coupling. Table 4 reports the influence of the piezoelectric coupling obtained with different theories. For a thin plate $(a / h=100)$, the differences between different formulations are rather small, except for first-order ESL models (ED1 and EDZ1): this clearly states the importance of the inclusion of the electrical stiffness, which cannot be represented by first-order formulations characterized by a linear distribution of the electric potential. Nonetheless, the linear layerwise formulation LD1 captures the relevant effects due to a localized adjusting of the mechanical and electrical field in each layer. In the case of a thick plate $(a / h=4)$, the error introduced by lower-order approximations in the mechanical field seems to be dominant: for example, considering the ED4 case, the error in the estimated influence of the piezoelectric effect is approximately $100 \%$ (3.02\% for ED4 compared to $1.50 \%$ for LD4), but the electric field model is exactly the same of the LD4 case, i.e. a layerwise, fourth-order model. In the case of first-order formulations, the piezoelectric influence results to be dramatically underestimated: The very large errors introduced by the unsatisfactory modeling of the mechanical field are in these cases superimposed to the errors deriving from the disregard of the electrical stiffness leading to a meaningless result.

In this work, the above case study is extended to curved shells by considering the free-vibration response of a sim-
Table 5

Influence of piezoelectric coupling on $\gamma_{2}=\omega_{2} \times 10^{-5}[\mathrm{rad} / \mathrm{s}]$ for a hollow, laminated piezoelectric cylinder $(a / h=10)$

\begin{tabular}{lllllll}
\hline & \multicolumn{6}{l}{$R_{\beta} / a$} \\
\cline { 2 - 6 } & 2 & 5 & 10 & 20 & 50 & 100 \\
\hline LD4 & 15.0880 & 10.0089 & 9.0537 & 8.7987 & 8.7260 & 8.7155 \\
LD4m & 14.7289 & 9.7321 & 8.7896 & 8.5377 & 8.4659 & 8.4555 \\
$\Delta[\%]$ & 2.38 & 2.76 & 2.92 & 2.97 & 2.98 & 2.98 \\
\hline
\end{tabular}

Same lay-up and materials of [32].

Table 6

Influence of piezoelectric coupling on $\gamma_{2}=\omega_{2} \times 10^{-5}[\mathrm{rad} / \mathrm{s}]$ for a hollow, laminated piezoelectric cylinder $\left(a / R_{\beta}=1\right)$

\begin{tabular}{lllllll}
\hline \multicolumn{7}{c}{$h / R_{\beta}$} \\
& 0.01 & 0.02 & 0.05 & 0.1 & 0.2 & 0.5 \\
\hline LD4 & 2.4779 & 4.9662 & 12.5864 & 26.1330 & 56.2050 & 154.3188 \\
LD4m & 2.4247 & 4.8594 & 12.3144 & 25.5693 & 55.0733 & 154.8242 \\
$\Delta[\%]$ & 2.15 & 2.15 & 2.16 & 2.16 & 2.01 & 1.62 \\
\hline
\end{tabular}

Same lay-up and materials of [32].

ply supported, hollow cylinder consisting of the same materials and lay-up of the plate. The free-vibration frequency parameter $\gamma_{2}=\omega_{2} \times 10^{-5}$ for the second axisymmetric mode is given. Note that the fundamental axisymmetric frequency corresponds to the mode obtained for $m=0$, $n=4$ but, since this mode is characterized by a vanishing deformation in thickness direction, i.e. $u_{z}^{k}=0$ in the whole cross-section, the piezoelectric effect on this mode can be neglected. The second frequency parameter $\gamma_{2}$ is associated to the "flexural" mode with $m=1, n=0$ and it involves a deformation in the thickness direction. In Table 5, results are given for different curvatures radii $R_{\beta}$ and for a constant thickness-to-length ratio $a / h=10$. On the other hand, in Table 6 the variable geometric parameter is the thickness while the curvature ratio $a / R_{\beta}$ has been kept constant and equal to 1 . In all cases, the electrical boundary conditions are a short-circuit between the external shell surfaces. In order to verify the influence of curvature and thickness on the electro-mechanical coupling in laminated piezoelectric shells, the data obtained by the most accurate LD4 formulation are reported for both the coupled and the uncoupled case.

Observing Table 6 it can be stated that the thickness of the hollow cylinder has a smaller effect on the electromechanical coupling with respect to the case of flat plate. The variation induced in the natural frequency by the piezoelectric effect is nearly constant for thin cylinders (about 2.15\%) and is reduced to about $1.6 \%$ for thick cylinders, whereas for the flat plate variations up to $4 \%$ could be established by varying the plate thickness. As far as the curvature is concerned, Table 5 shows a variation of the natural frequency of the selected mode ranging from $2.4 \%$ for small curvature radii up to $3 \%$ for 
shallow cylinders. Thus, a reduction of the curvature slightly increases the influence of the piezoelectric effect. From these results it appears that the electric stiffness has nearly a constant effect on the considered axisymmetric vibration mode, i.e. the geometric parameters do not modify significantly the role played by the electromechanical coupling.

\section{Conclusion}

This paper has presented a closed-form solution for the free-vibration problem of multilayered shells with embedded piezoelectric layers. The hierarchic modeling capability of the employed "Unified Formulation" allows the comparison between theories of different accuracy, ranging from a quasi-3D formulation to simple, lower-order theories. In particular, this work has focussed on the influence of the electro-mechanical coupling on the free-vibration response of multilayered structures. The relevance of the electric stiffness has been pointed out. Furthermore, a few parametric studies were presented in order to show the influence of some geometric parameters on the piezoelectric coupling effect. The thickness of a plate embedding thickness-polarized piezoelectric layers has shown to be a fundamental parameter: the piezoelectric coupling is more efficient in slender plates than in thick plates. For the considered vibration modes of hollow cylinders, the thickness and the curvature radius seem to have a less important role. The present work has shown that an accurate model for piezoelectric laminates can give important insights useful for the design of efficient piezoelectric devices. It is finally remarked that the adopted closed-form solution technique can be successfully used only for some specific cases; a more general solution method, based, e.g., on the Finite Element Method, could be employed in combination with the Unified Formulation to simulate more complicated structures.

\section{Appendix A. Fundamental nuclei of the formulation}

This appendix reports the explicit expressions for the fundamental nuclei appearing in Eqs. $(28,29)$. First, the following quantities are introduced from the integration in thickness direction over the layer thickness $h_{k}$ :

$$
\begin{aligned}
& \left(J^{k \tau s}, J_{\alpha / \beta}^{k \tau s}, J_{\beta / \alpha}^{k \tau s}, J_{\alpha \beta}^{k \tau s}\right)=\int_{h_{k}} F_{\tau} F_{s}\left(1, \frac{H_{\alpha_{k}}}{H_{\beta_{k}}}, \frac{H_{\beta_{k}}}{H_{\alpha_{k}}}, H_{\alpha_{k}} H_{\beta_{k}}\right) \mathrm{d} z_{k} \\
& \left(J_{\alpha}^{k \tau_{z} s}, J_{\beta}^{k \tau_{z} s}\right)=\int_{h_{k}} \frac{\partial F_{\tau}}{\partial z} F_{s}\left(H_{\alpha_{k}}, H_{\beta_{k}}\right) \mathrm{d} z_{k} \\
& \left(J_{\alpha}^{k \tau s_{z}}, J_{\beta}^{k \tau s_{z}}\right)=\int_{h_{k}} F_{\tau} \frac{\partial F_{s}}{\partial z}\left(H_{\alpha_{k}}, H_{\beta_{k}}\right) \mathrm{d} z_{k} \\
& J_{\alpha \beta}^{k \tau z_{z} s_{z}}=\int_{h_{k}} \frac{\partial F_{\tau}}{\partial z} \frac{\partial F_{s}}{\partial z} H_{\alpha_{k}} H_{\beta_{k}} \mathrm{~d} z_{k}
\end{aligned}
$$

Upon introduction of the above layer integrals, the fundamental arrays for the domain equations read:

$$
\begin{aligned}
& \left(K_{u u}^{\tau s k}\right)_{11}=-\widetilde{C}_{11}^{k} J_{\beta / \alpha}^{k \tau s} \partial_{\alpha \alpha}-2 \widetilde{C}_{16}^{k} J^{k \tau s} \partial_{\alpha \beta}-\widetilde{C}_{66}^{k} J_{\alpha / \beta}^{k \tau s} \partial_{\beta \beta} \\
& +\widetilde{C}_{55}^{k}\left(J_{\alpha \beta}^{k \tau_{z} s_{z}}-\lambda_{\mathrm{D}} \frac{1}{R_{\alpha_{k}}} J_{\beta}^{k \tau_{z} s}-\lambda_{\mathrm{D}} \frac{1}{R_{\alpha_{k}}} J_{\beta}^{k \tau s_{z}}+\lambda_{\mathrm{D}} \frac{1}{R_{\alpha_{k}}} \frac{1}{R_{\alpha_{k}}} J_{\beta / \alpha}^{k \tau s}\right) \\
& \left(K_{u u}^{\tau s k}\right)_{12}=-\widetilde{C}_{12}^{k} J^{k \tau s} \partial_{\alpha \beta}-\widetilde{C}_{16}^{k} J_{\beta / \alpha}^{k \tau s} \partial_{\alpha \alpha}-\widetilde{C}_{26}^{k} J_{\alpha / \beta}^{k \tau s} \partial_{\beta \beta}-\widetilde{C}_{66}^{k} J^{k \tau s} \partial_{\alpha \beta} \\
& +\widetilde{C}_{45}^{k}\left(J_{\alpha \beta}^{k \tau_{\beta} s_{z}}-\lambda_{\mathrm{D}} \frac{1}{R_{\beta_{k}}} J_{\alpha}^{k \tau_{z} s}-\lambda_{\mathrm{D}} \frac{1}{R_{\alpha_{k}}} J_{\beta}^{k \tau s_{z}}+\lambda_{\mathrm{D}} \frac{1}{R_{\alpha_{k}}} \frac{1}{R_{\beta_{k}}} J^{k \tau s}\right) \\
& \left(K_{u u}^{\tau s k}\right)_{13}=-\widetilde{C}_{11}^{k} \frac{1}{R_{\alpha_{k}}} J_{\beta / \alpha}^{k \tau s} \partial_{\alpha}-\widetilde{C}_{12}^{k} \frac{1}{R_{\beta_{k}}} J^{k \tau s} \partial_{\alpha}-\widetilde{C}_{13}^{k} J_{\beta}^{k \tau s_{z}} \partial_{\alpha} \\
& -\widetilde{C}_{16}^{k} \frac{1}{R_{\alpha_{k}}} J^{k \tau s} \partial_{\beta}-\widetilde{C}_{26}^{k} \frac{1}{R_{\beta_{k}}} J_{\alpha, \beta}^{k \tau s} \partial_{\beta}-\widetilde{C}_{36}^{k} J_{\alpha}^{k \tau s_{z}} \partial_{\beta} \\
& +\widetilde{C}_{45}^{k}\left(J_{\alpha}^{k \tau_{z} s} \partial_{\beta}-\lambda_{\mathrm{D}} \frac{1}{R_{\alpha_{k}}} J^{k \tau s} \partial_{\beta}\right)+\widetilde{C}_{55}^{k}\left(J_{\beta}^{k \tau_{z} s} \partial_{\alpha}-\lambda_{\mathrm{D}} \frac{1}{R_{\alpha_{k}}} J_{\beta / \alpha}^{k \tau s} \partial_{\alpha}\right) \\
& \left(K_{u u}^{\tau s k}\right)_{21}=-\widetilde{C}_{12}^{k} J^{k \tau s} \partial_{\alpha \beta}-\widetilde{C}_{16}^{k} J_{\beta / \alpha}^{k \tau s} \partial_{\alpha \alpha}-\widetilde{C}_{26}^{k} J_{\alpha / \beta}^{k \tau s} \partial_{\beta \beta}-\widetilde{C}_{66}^{k} J^{k \tau s} \partial_{\alpha \beta} \\
& +\widetilde{C}_{45}^{k}\left(J_{\alpha \beta}^{k \tau_{z} s_{z}}-\lambda_{\mathrm{D}} \frac{1}{R_{\beta_{k}}} J_{\alpha}^{k \tau s_{z}}-\lambda_{\mathrm{D}} \frac{1}{R_{\alpha_{k}}} J_{\beta}^{k \tau_{z} s}+\lambda_{\mathrm{D}} \frac{1}{R_{\alpha_{k}}} \frac{1}{R_{\beta_{k}}} J^{k \tau s}\right) \\
& \left(K_{u u}^{\tau s k}\right)_{22}=-\widetilde{C}_{22}^{k} J_{\alpha / \beta}^{k \tau s} \partial_{\beta \beta}-2 \widetilde{C}_{26}^{k} J^{k \tau s} \partial_{\alpha \beta}-\widetilde{C}_{66}^{k} J_{\beta / \alpha}^{k \tau s} \partial_{\alpha \alpha} \\
& +\widetilde{C}_{44}^{k}\left(J_{\alpha \beta}^{k \tau_{\tau} s_{z}}-\lambda_{\mathrm{D}} \frac{1}{R_{\beta_{k}}} J_{\alpha}^{k \tau_{z} s}-\lambda_{\mathrm{D}} \frac{1}{R_{\beta_{k}}} J_{\alpha}^{k \tau s_{z}}+\lambda_{\mathrm{D}} \frac{1}{R_{\beta_{k}}} \frac{1}{R_{\beta_{k}}} J_{\alpha / \beta}^{k \tau s}\right) \\
& \left(K_{u u}^{\tau s k}\right)_{23}=-\widetilde{C}_{12}^{k} \frac{1}{R_{\alpha_{k}}} J^{k \tau s} \partial_{\beta}-\widetilde{C}_{22}^{k} \frac{1}{R_{\beta_{k}}} J_{\alpha / \beta}^{k \tau s} \partial_{\beta}-\widetilde{C}_{23}^{k} J_{\alpha}^{k \tau s_{z}} \partial_{\beta} \\
& -\widetilde{C}_{16}^{k} \frac{1}{R_{\alpha_{k}}} J_{\beta / \alpha}^{k \tau s} \partial_{\alpha}-\widetilde{C}_{26}^{k} \frac{1}{R_{\beta_{k}}} J^{k \tau s} \partial_{\alpha}-\widetilde{C}_{36}^{k} J_{\beta}^{k \tau s_{z}} \partial_{\alpha} \\
& +\widetilde{C}_{45}^{k}\left(J_{\beta}^{k \tau_{z} s} \partial_{\alpha}-\lambda_{\mathrm{D}} \frac{1}{R_{\beta_{k}}} J^{k \tau s} \partial_{\alpha}\right)+\widetilde{C}_{44}^{k}\left(J_{\alpha}^{k \tau_{z} s} \partial_{\beta}-\lambda_{\mathrm{D}} \frac{1}{R_{\beta_{k}}} J_{\alpha / \beta}^{k \tau s} \partial_{\beta}\right) \\
& \left(K_{u u}^{\tau s k}\right)_{31}=\widetilde{C}_{11}^{k} \frac{1}{R_{\alpha_{k}}} J_{\beta / \alpha}^{k \tau s} \partial_{\alpha}+\widetilde{C}_{12}^{k} \frac{1}{R_{\beta_{k}}} J^{k \tau s} \partial_{\alpha}+\widetilde{C}_{13}^{k} J_{\beta}^{k \tau_{z} s} \partial_{\alpha} \\
& +\widetilde{C}_{16}^{k} \frac{1}{R_{\alpha_{k}}} J^{k \tau s} \partial_{\beta}+\widetilde{C}_{26}^{k} \frac{1}{R_{\beta_{k}}} J_{\alpha / \beta}^{k \tau s} \partial_{\beta}+\widetilde{C}_{36}^{k} J_{\alpha}^{k \tau_{z} s} \partial_{\beta} \\
& -\widetilde{C}_{45}^{k}\left(J_{\alpha}^{k \tau s_{z}} \partial_{\beta}-\lambda_{\mathrm{D}} \frac{1}{R_{\alpha_{k}}} J^{k \tau s} \partial_{\beta}\right)-\widetilde{C}_{55}^{k}\left(J_{\beta}^{k \tau s_{z}} \partial_{\alpha}-\lambda_{\mathrm{D}} \frac{1}{R_{\alpha_{k}}} J_{\beta / \alpha}^{k \tau s} \partial_{\alpha}\right) \\
& \left(K_{u u}^{\tau s k}\right)_{32}=\widetilde{C}_{12}^{k} \frac{1}{R_{\alpha_{k}}} J^{k \tau s} \partial_{\beta}+\widetilde{C}_{22}^{k} \frac{1}{R_{\beta_{k}}} J_{\alpha / \beta}^{k \tau s} \partial_{\beta}+\widetilde{C}_{23}^{k} J_{\alpha}^{k \tau_{z} s} \partial_{\beta} \\
& +\widetilde{C}_{16}^{k} \frac{1}{R_{\alpha_{k}}} J_{\beta / \alpha}^{k \tau s} \partial_{\alpha}+\widetilde{C}_{26}^{k} \frac{1}{R_{\beta_{k}}} J^{k \tau s} \partial_{\alpha}+\widetilde{C}_{36}^{k} J_{\beta}^{k \tau_{z} s} \partial_{\alpha} \\
& -\widetilde{C}_{45}^{k}\left(J_{\beta}^{k \tau s_{z}} \partial_{\alpha}-\lambda_{\mathrm{D}} \frac{1}{R_{\beta_{k}}} J^{k \tau s} \partial_{\alpha}\right)-\widetilde{C}_{44}^{k}\left(J_{\alpha}^{k \tau s_{z}} \partial_{\beta}-\lambda_{\mathrm{D}} \frac{1}{R_{\beta_{k}}} J_{\alpha / \beta}^{k \tau s} \partial_{\beta}\right) \\
& \left(K_{u u}^{\tau s k}\right)_{33}=\widetilde{C}_{11}^{k} \frac{1}{R_{\alpha_{k}}} \frac{1}{R_{\alpha_{k}}} J_{\beta / \alpha}^{k \tau s}+\widetilde{C}_{22}^{k} \frac{1}{R_{\beta_{k}}} \frac{1}{R_{\beta_{k}}} J_{\alpha / \beta}^{k \tau s}+\widetilde{C}_{33}^{k} J_{\alpha, \beta}^{k \tau \tau_{z} s_{z}} \\
& +2 \widetilde{C}_{12}^{k} \frac{1}{R_{\alpha_{k}}} \frac{1}{R_{\beta_{k}}} J^{k \tau s}+\widetilde{C}_{13}^{k} \frac{1}{R_{\alpha_{k}}}\left(J_{\beta}^{k \tau_{z} s}+J_{\beta}^{k \tau s_{z}}\right)+\widetilde{C}_{23}^{k} \frac{1}{R_{\beta_{k}}}\left(J_{\alpha}^{k \tau_{z} s}+J_{\alpha}^{k \tau s_{z}}\right) \\
& -\widetilde{C}_{44}^{k} J_{\alpha / \beta}^{k \tau s} \partial_{\beta \beta}-\widetilde{C}_{55}^{k} J_{\beta / \alpha}^{k \tau s} \partial_{\alpha \alpha}-2 \widetilde{C}_{45}^{k} J^{k \tau s} \partial_{\alpha \beta} \\
& \left(K_{u e}^{\tau s k}\right)_{11}=e_{15}\left(J_{\beta}^{k \tau_{z} s}-\lambda_{\mathrm{D}} \frac{1}{R_{\alpha_{k}}} J_{\beta / \alpha}^{k \tau s}\right) \partial_{\alpha} \\
& \left(K_{u e}^{\tau s k}\right)_{12}=e_{14}\left(J_{\alpha}^{k \tau_{z} s}-\lambda_{\mathrm{D}} \frac{1}{R_{\alpha_{k}}} J^{k \tau s}\right) \partial_{\beta} \\
& \left(K_{u e}^{\tau s k}\right)_{13}=-e_{31} J_{\beta}^{k \tau s_{z}} \partial_{\alpha}-e_{36} J_{\alpha}^{k \tau s_{z}} \partial_{\beta} \\
& \left(K_{u e}^{\tau s k}\right)_{21}=e_{25}\left(J_{\beta}^{k \tau_{z} s}-\lambda_{\mathrm{D}} \frac{1}{R_{\beta_{k}}} J^{k \tau s}\right) \partial_{\alpha} \\
& \left(K_{u e}^{\tau s k}\right)_{22}=e_{24}\left(J_{\alpha}^{k \tau_{z} s}-\lambda_{\mathrm{D}} \frac{1}{R_{\beta_{k}}} J_{\alpha / \beta}^{k \tau s}\right) \partial_{\beta} \\
& \left(K_{u e}^{\tau s k}\right)_{23}=-e_{32} J_{\alpha}^{k \tau s_{z}} \partial_{\beta}-e_{36} J_{\beta}^{k \tau s_{z}} \partial_{\alpha}
\end{aligned}
$$




$$
\begin{aligned}
& \left(K_{u e}^{\tau s k}\right)_{31}=-e_{15} J_{\beta / \alpha}^{k \tau s} \partial_{\alpha \alpha}-e_{25} J^{k \tau s} \partial_{\alpha \beta} \\
& \left(K_{u e}^{\tau s k}\right)_{32}=-e_{24} J_{\alpha / \beta}^{k \tau s} \partial_{\beta \beta}-e_{14} J^{k \tau s} \partial_{\alpha \beta} \\
& \left(K_{u e}^{\tau s k}\right)_{33}=e_{31} \frac{1}{R_{\alpha_{k}}} J_{\beta}^{k \tau s_{z}}+e_{32} \frac{1}{R_{\beta_{k}}} J_{\alpha}^{k \tau s_{z}}+e_{33} J_{\alpha \beta}^{k \tau_{z} s_{z}} \\
& \left(M^{\tau s k}\right)_{11}=\rho^{k} J_{\alpha \beta}^{k \tau s} ; \quad\left(M^{\tau s k}\right)_{22}=\rho^{k} J_{\alpha \beta}^{k \tau s} ; \quad\left(M^{\tau s k}\right)_{33}=\rho^{k} J_{\alpha \beta}^{k \tau s} \\
& \left(K_{e u}^{\tau s k}\right)_{11}=-e_{15}\left(J_{\beta}^{k \tau s_{z}}-\lambda_{\mathrm{D}} \frac{1}{R_{\alpha_{k}}} J_{\beta / \alpha}^{k \tau s}\right) \partial_{\alpha} \\
& \left(K_{e u}^{\tau s k}\right)_{12}=-e_{25}\left(J_{\beta}^{k \tau s_{z}}-\lambda_{\mathrm{D}} \frac{1}{R_{\beta_{k}}} J^{k \tau s}\right) \partial_{\alpha} \\
& \left(K_{e u}^{\tau s k}\right)_{13}=-e_{15} J_{\beta / \alpha}^{k \tau s} \partial_{\alpha \alpha}-e_{25} J^{k \tau s} \partial_{\alpha \beta} \\
& \left(K_{e u}^{\tau s k}\right)_{21}=-e_{14}\left(J_{\alpha}^{k \tau s_{z}}-\lambda_{\mathrm{D}} \frac{1}{R_{\alpha_{k}}} J^{k \tau s}\right) \partial_{\beta} \\
& \left(K_{e u}^{\tau s k}\right)_{22}=-e_{24}\left(J_{\alpha}^{k \tau s_{z}}-\lambda_{\mathrm{D}} \frac{1}{R_{\beta_{k}}} J_{\alpha / \beta}^{k \tau s}\right) \partial_{\beta} \\
& \left(K_{e u}^{\tau s k}\right)_{23}=-e_{24} J_{\alpha / \beta}^{k \tau s} \partial_{\beta \beta}-e_{14} J^{k \tau s} \partial_{\alpha \beta} \\
& \left(K_{e u}^{\tau s k}\right)_{31}=e_{31} J_{\beta}^{k \tau_{z} s} \partial_{\alpha}+e_{36} J_{\alpha}^{k \tau_{z} s} \partial_{\beta} \\
& \left(K_{e u}^{\tau s k}\right)_{32}=e_{32} J_{\alpha}^{k \tau_{z} s} \partial_{\beta}+e_{36} J_{\beta}^{k \tau_{z} s} \partial_{\alpha} \\
& \left(K_{e u}^{\tau s k}\right)_{33}=e_{31} \frac{1}{R_{\alpha_{k}}} J_{\beta}^{k \tau_{z} s}+e_{32} \frac{1}{R_{\beta_{k}}} J_{\alpha}^{k \tau_{z} s}+e_{33} J_{\alpha \beta}^{k \tau_{z} s_{z}} \\
& \left(K_{e e}^{\tau s k}\right)_{11}=\varepsilon_{11} J_{\beta / \alpha}^{k \tau s} \partial_{\alpha \alpha} \\
& \left(K_{e e}^{\tau s k}\right)_{22}=\varepsilon_{22} J_{\alpha / \beta}^{k \tau s} \partial_{\beta \beta} \\
& \left(K_{e e}^{\tau s k}\right)_{33}=-\varepsilon_{33} J_{\alpha \beta}^{k \tau_{z} s_{z}}
\end{aligned}
$$

For brevity, only the non-vanishing terms of the arrays have been quoted. Note, once the integration-by-parts has been performed, the in-plane differential operators $\partial_{\alpha}, \partial_{\beta}, \ldots, \partial_{\alpha \beta}$ act simply on the unknowns.

Finally, the fundamental nuclei related to the boundary terms read:

$$
\begin{aligned}
\left(\Pi_{u u}^{\tau s k}\right)_{11}= & \widetilde{C}_{11}^{k} J_{\beta / \alpha}^{k \tau s} \partial_{\alpha}+\widetilde{C}_{16}^{k} J^{k \tau s}\left(\partial_{\alpha}+\partial_{\beta}\right)+\widetilde{C}_{66}^{k} J_{\alpha / \beta}^{k \tau s} \partial_{\beta} \\
\left(\Pi_{u u}^{\tau s k}\right)_{12}= & \widetilde{C}_{12}^{k} J^{k \tau s} \partial_{\beta}+\widetilde{C}_{16}^{k} J_{\beta / \alpha}^{k \tau s} \partial_{\alpha}+\widetilde{C}_{26}^{k} J_{\alpha / \beta}^{k \tau s} \partial_{\beta}+\widetilde{C}_{66}^{k} J^{k \tau s} \partial_{\alpha} \\
\left(\Pi_{u u}^{\tau s k}\right)_{13}= & \widetilde{C}_{13}^{k} J_{\beta}^{k \tau s_{z}}+\widetilde{C}_{36}^{k} J_{\alpha}^{k \tau s_{z}}+\frac{1}{R_{\alpha_{k}}}\left(\widetilde{C}_{11}^{k} J_{\beta / \alpha}^{k \tau s}+\widetilde{C}_{16}^{k} J^{k \tau s}\right) \\
& +\frac{1}{R_{\beta_{k}}}\left(\widetilde{C}_{26}^{k} J_{\alpha / \beta}^{k \tau s}+\widetilde{C}_{12}^{k} J^{k \tau s}\right) \\
\left(\Pi_{u u}^{\tau s k}\right)_{21}= & \widetilde{C}_{12}^{k} J^{k \tau s} \partial_{\alpha}+\widetilde{C}_{16}^{k} J_{\beta / \alpha}^{k \tau s} \partial_{\alpha}+\widetilde{C}_{26}^{k} J_{\alpha / \beta}^{k \tau s} \partial_{\beta}+\widetilde{C}_{66}^{k} J^{k \tau s} \partial_{\beta} \\
\left(\Pi_{u u}^{\tau s k}\right)_{22}= & \widetilde{C}_{22}^{k} J_{\alpha / \beta}^{k \tau s} \partial_{\beta}+\widetilde{C}_{26}^{k} J^{k \tau s}\left(\partial_{\alpha}+\partial_{\beta}\right)+\widetilde{C}_{66}^{k} J_{\beta / \alpha}^{k \tau s} \partial_{\alpha} \\
\left(\Pi_{u u}^{\tau s k}\right)_{23}= & \widetilde{C}_{23}^{k} J_{\alpha}^{k \tau s_{z}}+\widetilde{C}_{36}^{k} J_{\beta}^{k \tau s_{z}}+\frac{1}{R_{\alpha_{k}}}\left(\widetilde{C}_{16}^{k} J_{\beta / \alpha}^{k \tau s}+\widetilde{C}_{12}^{k} J^{k \tau s}\right) \\
& +\frac{1}{R_{\beta_{k}}}\left(\widetilde{C}_{22}^{k} J_{\alpha / \beta}^{k \tau s}+\widetilde{C}_{26}^{k} J^{k \tau s}\right) \\
\left(\Pi_{u u}^{\tau s k}\right)_{31}= & \widetilde{C}_{55}^{k} J_{\beta}^{k \tau s_{z}}+\widetilde{C}_{45}^{k} J_{\alpha}^{k \tau s_{z}}-\lambda_{\mathrm{D}} \frac{1}{R_{\alpha_{k}}}\left(\widetilde{C}_{55}^{k} J_{\beta / \alpha}^{k \tau s}+\widetilde{C}_{45}^{k} J^{k \tau s}\right) \\
\left(\Pi_{u u}^{\tau s k}\right)_{32}= & \widetilde{C}_{44}^{k} J_{\alpha}^{k \tau s_{z}}+\widetilde{C}_{45}^{k} J_{\beta}^{k \tau s_{z}}-\lambda_{\mathrm{D}} \frac{1}{R_{\beta_{k}}}\left(\widetilde{C}_{44}^{k} J_{\alpha / \beta}^{k \tau s}+\widetilde{C}_{45}^{k} J^{k \tau s}\right) \\
\left(\Pi_{u u}^{\tau s k}\right)_{33}= & \widetilde{C}_{44}^{k} J_{\alpha / \beta}^{k \tau s} \partial_{\beta}+\widetilde{C}_{45}^{k} J^{k \tau s}\left(\partial_{\alpha}+\partial_{\beta}\right)+\widetilde{C}_{55}^{k} J_{\beta / \alpha}^{k \tau s} \partial_{\alpha}
\end{aligned}
$$

$$
\begin{aligned}
& \left(\Pi_{u e}^{\tau s k}\right)_{13}=e_{31} J_{\beta}^{k \tau s_{z}}+e_{36} J_{\alpha}^{k \tau s_{z}} ; \quad\left(\Pi_{u e}^{\tau s k}\right)_{31}=e_{15} J_{\beta / \alpha}^{k \tau s} \partial_{\alpha}+e_{25} J^{k \tau s} \partial_{\alpha} \\
& \left(\Pi_{u e}^{\tau s k}\right)_{23}=e_{32} J_{\alpha}^{k \tau s_{z}}+e_{36} J_{\beta}^{k \tau s_{z}} ; \quad\left(\Pi_{u e}^{\tau s k}\right)_{32}=e_{24} J_{\alpha / \beta}^{k \tau s} \partial_{\beta}+e_{14} J^{k \tau s} \partial_{\beta} \\
& \left(\Pi_{e u}^{\tau s k}\right)_{11}=e_{15}\left(J_{\beta}^{k \tau s_{z}}-\lambda_{\mathrm{D}} \frac{1}{R_{\alpha_{k}}} J_{\beta / \alpha}^{k \tau s}\right) ; \quad\left(\Pi_{e u}^{\tau s k}\right)_{21}=e_{14}\left(J_{\alpha}^{k \tau s_{z}}-\lambda_{\mathrm{D}} \frac{1}{R_{\alpha_{k}}} J^{k \tau s}\right) \\
& \left(\Pi_{e u}^{\tau s k}\right)_{12}=e_{25}\left(J_{\beta}^{k \tau s_{z}}-\lambda_{\mathrm{D}} \frac{1}{R_{\beta_{k}}} J^{k \tau s}\right) ; \quad\left(\Pi_{e u}^{\tau s k}\right)_{22}=e_{24}\left(J_{\alpha}^{k \tau s_{z}}-\lambda_{\mathrm{D}} \frac{1}{R_{\beta_{k}}} J_{\alpha / \beta}^{k \tau s}\right) \\
& \left(\Pi_{e u}^{\tau s k}\right)_{13}=e_{15} J_{\beta / \alpha}^{k \tau s} \partial_{\alpha}+e_{25} J^{k \tau s} \partial_{\beta} ; \quad\left(\Pi_{e u}^{\tau s k}\right)_{23}=e_{24} J_{\alpha / \beta}^{k \tau s} \partial_{\beta}+e_{14} J^{k \tau s} \partial_{\alpha} \\
& \left(\Pi_{e e}^{\tau s k}\right)_{11}=-\varepsilon_{11} J_{\beta / \alpha}^{k \tau s} \partial_{\alpha} ; \quad\left(\Pi_{e e}^{\tau s k}\right)_{22}=-\varepsilon_{22} J_{\alpha / \beta}^{k \tau s} \partial_{\beta}
\end{aligned}
$$

\section{References}

[1] Noor AK, Burton WS. Assessment of computational models for multilayered shells. Appl Mech Rev 1990;43(4):67-97.

[2] Carrera E. Historical review of zig-zag theories for multilayered plates and shells. Appl Mech Rev 2003;56(3):287-308.

[3] Reddy JN. Mechanics of laminated composite plates and shells: theory and analysis. Boca Raton: CRC Press; 2004.

[4] Carrera E. $C_{z}^{0}$ requirements - models for the two dimensional analysis of multilayered structures. Compos Struct 1997;37:373-83.

[5] Koiter WT. A consistent first approximation in the general theory of thin elastic shells. In: Koiter WT, editor. The theory of thin elastic shells. Delft: IUTAM, North-Holland; 1959. p. 1233.

[6] Mindlin RD. Forced thickness-shear and flexural vibrations of piezoelectric crystal plates. J Appl Phys 1952;23:83-8.

[7] EerNisse EP. Variational method for electroelastic vibration analysis. IEEE Trans Sonics Ultrasonics 1967;SU-14(4):153-60.

[8] Tiersten HF, Mindlin RD. Forced vibrations of piezoelectric crystal plates. Quart Appl Math 1962;20(2):107-19.

[9] Tiersten HF. Linear piezoelectric plate vibrations. New York: Plenum; 1969.

[10] Saravanos DA, Heyliger PR. Mechanics and computational models for laminated piezoelectric beams, plates and shells. Appl Mech Rev 1999;52(10):305-19.

[11] Kapuria S. A coupled zig-zag third-order theory for piezoelectric hybrid cross-ply plates. J Appl Mech 2004;71:604-14.

[12] Ossadzow-David C, Touratier M. A multilayered piezoelectric shell theory. Compos Sci Technol 2004;64:2121-37.

[13] Heyliger P, Pei KC, Saravanos DA. Layerwise mechanics and finite element model for laminated piezoelectric shells. AIAA J 1996;34(11):2353-60.

[14] Rogacheva NN. The theory of piezoelectric shells and plates. Boca Raton: CRC Press; 1994.

[15] Gopinathan SV, Varadan VV, Varadan VK. A review and critique of theories for piezoelectric laminates. Smart Mater Struct 2000;9:2448.

[16] Benjeddou A. Advances in piezoelectric finite element modeling of adaptive structural elements: a survey. Comput Struct 2000;76: $347-63$.

[17] Lammering R, Mesecke-Rischmann S. Multi-field variational formulations and related finite elements for piezoelectric shells. Smart Mater Struct 2003;12(6):904-13.

[18] Benjeddou A, Andrianarison O. Extension of Reissner's mixed variational theorem to piezoelectric multilayered composites. In: Lipitakis EA, editor. Proceedings of the sixth Hellenic-European conference on computer mathematics and its applications, LEA, Athens, Hellas, 2003. p. 116-24.

[19] Carrera E. Theories and finite elements for multilayered plates and shells: A unified compact formulation with numerical assessment and benchmarking. Arch Comput Methods Eng 2003;10(3):21596.

[20] Carrera E. Single-layer vs multilayer plate modelings on the base of Reissner's mixed theorem. AIAA J 2000;38(2):342-52. 
[21] Carrera E. Multilayered shell theories accounting for layerwise mixed description. Part 1: Governing equations; Part 2: Numerical evaluations. AIAA J 1999;37(9):1107-24.

[22] Ballhause D, D’Ottavio M, Kröplin B, Carrera E. A unified formulation to assess multilayered theories for piezoelectric plates. Comput Struct 2005;83(15-16):1217-35.

[23] Kraus H. Thin elastic shells. John Wiley \& Sons, Inc.; 1967.

[24] Novozhilov VV. Thin shell theory. 2nd ed. Groningem: P. Noordhoff Ltd.; 1964

[25] Ikeda T. Fundamental of piezoelectricity. Oxford University Press; 1996.

[26] Murakami H. Laminated composite plate theory with improved inplane response. J Appl Mech 1986;53:661-6.

[27] Carrera E. On the use of Murakami's zig-zag function in the modeling of layered plates and shells. Comput Struct 2004;82:541-54.
[28] Carrera E. A class of two dimensional theories for multilayered plates analysis. Atti Acc Sci Torino, Mem Sci Fis 1995;19-20:4987.

[29] Carrera E. A study of transverse normal stress effect on vibration of multilayered plates and shells. J Sound Vib 1999;225(5):803-29.

[30] Drumheller DS, Kalnins A. Dynamic shell theory for ferroelectric ceramics. J Acoust Soc Am 1970;47:1343-53.

[31] Benjeddou A. Modelling and simulation of adaptive structures and composites: Current trends and future directions. In: Topping BHV, Mota-Soares CA, editors. Progress in computational structures technology. Stirling, UK: Saxe-Coburg Publications; 2004. p. 251-80.

[32] Heyliger PR, Saravanos DA. Exact free-vibration analysis of laminated plates with embedded piezoelectric layers. J Acoust Soc Am 1995;98(3):1547-57. 